SUBMETIDO EM 11/06/2014. ACEITO EM 30/03/2015.

\title{
Gráfico T2 de Hotelling com tamanho de amostra variável aplicado ao processo de produção de itens de segurança automotiva
}

\author{
Raphael Henrique Teixeira da Silva \\ Universidade Federal de São João del Rei \\ raphaelhts@hotmail.com
}

\author{
Daniela Carine Ramires de Oliveira \\ Universidade Federal de São João del Rei \\ ramires.daniela@gmail.com
}

\section{RESUMO}

Visitas técnicas a empresas da região de São João del Rei-MG têm mostrado que o monitoramento de características do processo tem sido realizado com gráficos de controle de qualidade univariados. No entanto, esses processos analisam várias variáveis, as mesmas usualmente estão correlacionadas e, a literatura mostra que analisar separadamente não é adequado. Além disso, verifica-se na rotina desses processos o uso de tamanho de amostra variável. A teoria e implementação computacional das versões multivariadas dos gráficos de controle são apresentadas, em geral, quando o tamanho amostral é fixo. Devido a dificuldade de se obter essas duas informações simultaneamente na literatura, as empresas apresentam resistência ao uso dessas versões multivariadas para o controle de qualidade de seus produtos. Nesse contexto, esse trabalho apresenta a teoria e implementação computacional no software livre $\mathrm{R}$ do gráfico $\mathrm{T}^{2}$ de Hotelling, quando o tamanho da amostra é variável. Adicionalmente, é apresentado um estudo de caso.

Palavras-chave: Limite superior de controle variado; Controle estatístico de qualidade; Gráfico de controle multivariado.

\begin{abstract}
Technical visits to companies in the region of São João del Rei-MG have shown that the characteristics of the monitoring process are being carried out using univariate control charts. However, these processes analyze multiple variables, they are usually correlated, and the literature shows that it is not appropriate to analyze separately. Furthermore, we may verify variable sample size in the routine use of these processes. The theory and computational implementation of multivariate versions of the control charts are presented mostly when the sample size is fixed. Due to the difficulty of getting those two informations simultaneously in the literature, factories are resistant to the use of these multivariate versions for the quality control of their products. In this context, this paper presents the theory and computational implementation in the software R of the Hotelling $\mathrm{T}^{2}$ control chart when the sample size is variable. Additionally, a case study is presented.
\end{abstract}


Keywords: Variable upper control limit; Statistical quality control; Multivariate control chart.

\section{Introdução}

A competitividade existente no mercado é um fator cada vez mais presente na realidade das empresas. Para tanto, pode-se dizer que o controle estatístico da qualidade é uma forma eficaz para se controlar um processo, garantindo a melhoria contínua da produção. Entre as principais ferramentas do controle estatístico de qualidade, os gráficos de controle se destacam. O principal objetivo desses gráficos é detectar causas especiais (inaceitáveis) existentes em um processo. Os gráficos de controle univariados são os mais comuns na realidade das empresas e servem de grande auxílio para o monitoramento on-line da produção. Entretanto, com os processos industriais da região de São João del Rei-MG, esses gráficos, em geral, não têm se mostrado eficientes. Para as situações encontradas, os gráficos de controle multivariados mostraram ser mais adequados, uma vez que realizam o monitoramento de mais de uma característica de qualidade de forma simultânea e, as mesmas estão correlacionadas. A literatura indica que um possível gráfico de controle multivariado é o $\mathrm{T}^{2}$ de Hotelling.

$\mathrm{O} \mathrm{T}^{2}$ de Hotelling tem sido usado em aplicações de processos de diversos segmentos. Alencar, Lopes e Souza (2007) utilizaram para verificar o desempenho do processo de compressão de comprimidos de captopril, avaliando simultaneamente as variáveis: peso médio, dureza, friabilidade, desintegração, teor, teor de dissulfeto de captopril, dissolução e uniformidade de conteúdo dos comprimidos. Follador (2010) o utilizou para verificar a qualidade da água a montante e a jusante do rio Mandurim, avaliando conjuntamente as variáveis: $\mathrm{pH}$, oxigênio dissolvido, fósforo, nitrogênio, demanda bioquímica de oxigênio, coliformes termotolerantes, temperatura, turbidez e sólidos. Oyeyemi (2011) o apresenta para o controle do processo de produção de um detergente, considerando as características: detergente ativo, teor de umidade, densidade e nível de pH. Rojas et al. (2012) utilizaram para avaliar a diferenciação morfométrica entre duas espécies parasitas de cabras, medindo: o comprimento de pico, a distância do gancho de esquerda e distância do gancho de direita; essas variáveis são referentes às dimensões das espículas das espécies em estudo. Singh e Kumar (2011) utilizaram para comparar a qualidade da água de duas estações de monitoramento, avaliando as variáveis: cloreto, alcalinidade, flúor e sólidos dissolvidos totais. Melo (2008) aplicou-o para o controle de processos internos de agências bancárias, avaliando: operações de crédito contratadas, cadastro e limite de crédito, contábil e gerenciamento de fluxo de numerário. Lima et al. (2012) utilizaram para aperfeiçoar o processo de monitorar e controlar a qualidade de uma empresa que produz garrafas plásticas, avaliando as características: comprimento externo, comprimento interno, largura externa, largura interna, altura total, altura de empilhamento, comprimento de fundo, largura de fundo, altura do ninho (altura das divisórias internas), altura de encaixe do fundo. Em todas as citações mencionadas, cabe ressaltar que o tamanho amostral era fixo.

Nesse contexto, devido a vários estudos relacionados ao gráfico $\mathrm{T}^{2}$ de Hotelling não apresentarem o tamanho de amostra variável, o objetivo deste trabalho foi apresentar a teoria proposta por Hotelling com tamanho de amostra variável e a implementação computacional no software livre $\mathrm{R}$ versão 3.1.0, como também, realizar um estudo de caso em uma empresa que produz itens de segurança para automóveis que está situada na região de São João del Rei - MG.

\section{Revisão bibliográfica}

Nesta seção, são apresentados os conceitos básicos sobre cada um dos tópicos a seguir, com o objetivo de uniformizar o conhecimento sobre: gráficos de controle univariados, quando o tamanho da amostra é variável; distribuição normal multivariada e teste de hipóteses para a verificação da normalidade multivariada; coeficiente de correlação e teste de hipóteses para a verificação de sua significância; vetor de médias e matriz de variâncias e covariâncias amostrais; gráfico de controle multivariado, quando o tamanho da amostra é variável e a decomposição da estatística $\mathrm{T}^{2}$ de Hotelling.

\subsection{Conceitos sobre gráficos de controle}


Os gráficos de controle são ferramentas estatísticas para o monitoramento de processos. Estes gráficos são eficazes para a melhoria contínua da qualidade. A literatura apresenta gráficos de controle que monitoram uma característica da qualidade de cada vez (gráficos univariados) e os que monitoram mais de uma característica da qualidade de forma simultânea (gráficos multivariados) (COSTA, EPPRECHT \& CARPINETTI, 2012).

Um gráfico de controle é utilizado para verificar se um processo está sob controle estatístico com o auxílio de seus Limites de Controle. O mesmo consiste em um Limite Superior de Controle (LSC), um Limite Inferior de Controle (LIC) e uma Linha Central (LC). Esses limites são escolhidos de modo que, se o processo estiver sob controle, praticamente todos os pontos amostrais estarão entre os limites. No entanto, um ponto que caia fora dos limites de controle é interpretado como evidência de que o processo está fora de controle. Neste caso, investigação e ação corretiva serão necessárias para encontrar e eliminar a causa ou as causas especiais responsáveis por esse comportamento (SAMOHYL, 2009).

Segundo Montgomery (2009), mesmo que todos os pontos estejam dentro dos limites de controle, se houver um comportamento cíclico ou não aleatório, isso pode evidenciar um processo fora de controle. Existem alguns critérios de utilização de gráficos de controle para determinar se um processo está fora de controle estatístico (ver Tabela 1).

Tabela 1 - Critérios para identificação de causas especiais em um processo

Resumo dos Critérios de utilização de gráficos de controle

\begin{tabular}{lc}
\hline 1 & Um ou mais pontos fora dos limites três sigma \\
2 & Nove pontos seguidos acima ou abaixo da linha central \\
3 & Seis pontos seguidos, todos crescentes ou decrescentes \\
4 & Quatorze pontos seguidos alternados para cima e para baixo \\
5 & Dois de três pontos fora dos limites dois sigma \\
6 & Quatro de cinco pontos fora dos limites um sigma \\
7 & Quinze pontos seguidos dentro de um dos intervalos um sigma \\
8 & Oito pontos seguidos fora do limite um sigma \\
\hline
\end{tabular}

Fonte: Montgomery (2009).

\subsection{Gráficos de controle univariados para tamanho amostral variável}

Os gráficos de controle mais empregados para monitorar uma característica da qualidade numérica são os gráficos Xbarra (monitorando o valor médio) e o gráfico $\mathrm{S}$ (monitorando a variabilidade). Para aplicação dos gráficos univariados, a característica de qualidade deverá ser normalmente distribuída. Em sua maioria, a média populacional $\mu$ e o desvio padrão $\sigma$ não são conhecidos, então os mesmos devem ser estimados a partir de amostras ou subgrupos preliminares, retirados quando o processo está supostamente sob controle estatístico (SAMOHYL, 2009).

Os gráficos Xbarra e $\mathrm{S}$ são construídos, supondo que $m$ amostras estejam disponíveis, cada uma com um número de observações diferentes, representadas por $n_{k}$. Assim, o melhor estimador de $\mu$, a média do processo, é

$$
\overline{\bar{X}}=\frac{\sum_{\mathrm{k}=1}^{\mathrm{m}} \mathrm{n}_{\mathrm{k}} \overline{\mathrm{X}}_{\mathrm{k}}}{\sum_{\mathrm{k}=1}^{\mathrm{m}} \mathrm{n}_{\mathrm{k}}}
$$

onde $\overline{\mathrm{X}}_{\mathrm{k}}$ é a média das $n_{k}$ observações no instante $k$, com $\mathrm{k}=1, \ldots, \mathrm{m}$.

No gráfico Xbarra, $\overline{\bar{X}}$ é a linha central. Já para a linha central no gráfico $S$ é utilizada a seguinte expressão

$$
\overline{\mathrm{S}}=\left[\frac{\sum_{\mathrm{k}=1}^{\mathrm{m}}\left(\mathrm{n}_{\mathrm{k}}-1\right) \mathrm{S}_{\mathrm{k}}^{2}}{\left.\sum_{\mathrm{k}=1}^{\mathrm{m}} \mathrm{n}_{\mathrm{k}}-1\right)}\right]^{1 / 2}
$$

em que $\mathrm{S}_{\mathrm{k}}^{2}=\sum_{\mathrm{i}=1}^{\mathrm{n}_{\mathrm{k}}}\left(\mathrm{X}_{\mathrm{i}}-\overline{\mathrm{X}}_{\mathrm{k}}\right)^{2} /\left(\mathrm{n}_{\mathrm{k}}-1\right)$ é a variância amostral das $n_{k}$ observações no instante $k$, com $\mathrm{k}=1$, ..., $\mathrm{m}$.

Após definido os conceitos de média e desvio padrão para realização dos gráficos univariados, segue as fórmulas para a construção dos limites de controle. Para o gráfico Xbarra, no qual os pontos plotados são referentes às médias de cada amostra, os limites de controle são dados por: 


$$
\begin{aligned}
& \mathrm{LSC}_{\mathrm{k}}=\overline{\overline{\mathrm{X}}}+\mathrm{A}_{3} \overline{\mathrm{S}} \\
& \mathrm{LC}=\overline{\overline{\mathrm{X}}} \\
& \mathrm{LIC}_{\mathrm{k}}=\overline{\overline{\mathrm{X}}}-\mathrm{A}_{3} \overline{\mathrm{S}}
\end{aligned}
$$

A constante $A_{3}$ é um valor tabelado obtido de Montgomery (2009, Tabela VI, pg. 702), para vários tamanhos de amostra.

$\mathrm{O}$ gráfico $\mathrm{S}$ é usado para monitorar a variabilidade do processo, plotando-se os valores dos desvios padrões amostrais $S_{k}, k=1,2, \ldots, m$, onde $S_{k}$ é a raiz positiva de $S_{k}^{2}$, apresentado logo após a expressão (2). A linha central e os limites de controle para o gráfico $\mathrm{S}$ são dados por

$$
\begin{aligned}
& \mathrm{LSC}_{\mathrm{k}}=\mathrm{B}_{4} \overline{\mathrm{S}} \\
& \mathrm{LC}=\overline{\mathrm{S}} \\
& \mathrm{LIC}_{\mathrm{k}}=\mathrm{B}_{3} \overline{\mathrm{S}}
\end{aligned}
$$

As constantes $\mathrm{B}_{3}$ e $\mathrm{B}_{4}$ são valores tabelados, para vários tamanhos de amostra, obtidos de Montgomery (2009, Tabela VI, pg. 702).

\subsection{Distribuição normal multivariada e verificação das suposições}

O gráfico de controle multivariado que será apresentado a seguir possui a suposição de que as características da qualidade sigam uma distribuição normal multivariada. A expressão da distribuição normal multivariada é considerada uma forma generalizada da distribuição normal univariada.

A função densidade de probabilidade da distribuição normal univariada é definida por

$$
f(X)=\frac{1}{\sqrt{2 \pi \sigma^{2}}} e^{-\frac{1}{2}\left(\frac{X-\mu}{\sigma}\right)^{2}} \quad-\infty<X<\infty .
$$

A média da distribuição normal é $\mu$ e a variância é $\sigma^{2}$. Percebe-se que o termo no expoente da distribuição normal univariada em (5) pode ser escrito como:

$$
(X-\mu)\left(\sigma^{2}\right)^{-1}(X-\mu) \text {. }
$$
média $\mu$.

Essa quantidade mede a distância expressa em unidades de desvio padrão ao quadrado de $X$ à

Para a situação multivariada o caso descrito acima é análogo. Suponha que tenhamos $p$ variáveis, dadas por $X_{1}, X_{2}, \ldots, X_{p}$. Essas variáveis são arranjadas em um vetor de $p$ componentes $X^{\prime}=\left[X_{1}, X_{2}, \ldots, X_{p}\right]$. Seja $\mu^{\prime}=\left[\mu_{1}, \mu_{2}, \ldots, \mu_{p}\right]$ o vetor das médias dos $X$ 's, e considere as variâncias e as covariâncias das variáveis aleatórias em $X$ contidas em uma matriz de covariância $\sum$ de dimensão $p \times p$. Os elementos da diagonal principal de $\sum$ são as variâncias dos $X$ 's e os elementos fora da diagonal são as covariâncias. Agora, a distância (generalizada) padronizada ao quadrado de $X$ a $\mu$ é:

$$
(X-\mu)^{\prime} \sum^{-1}(X-\mu) \text {. }
$$

Isto é, obtém-se a função densidade de probabilidade da distribuição normal multivariada pela substituição da distância padronizada na expressão (6) pela distância generalizada multivariada da expressão (7), e pela mudança no termo constante $\frac{1}{\sqrt{2 \pi \sigma^{2}}}$ para uma forma mais geral que torne a área sob a função densidade de probabilidade unitária, independentemente do valor de $p$. Portanto, a função de densidade de probabilidade multivariada é

$$
f(X)=\frac{1}{(2 \pi)^{p / 2}|\Sigma|^{1 / 2}} e^{-\frac{1}{2}(X-\mu)^{\prime} \Sigma^{-1}(X-\mu)},
$$

em que $-\infty<X_{i}<\infty, \mathrm{i}=1,2, \ldots, p$.

Como foram abordadas duas características da qualidade $(p=2)$, foi verificada a suposição da distribuição normal bivariada, dada pela expressão (8), isto é, a função densidade de um vetor $X^{\prime}=\left[X_{1}, X_{2}\right]$ é definida por

$$
f\left(X_{1}, X_{2}\right)=\frac{1}{(2 \pi)|\Sigma|^{\frac{1}{2}}} e^{-\frac{1}{2}(X-\mu)^{\prime} \Sigma^{-1}(X-\mu)},
$$

em que $\mu$ é o vetor de médias e $\sum$ é a matriz de variâncias e covariâncias que pode ser obtida desta maneira

onde

$$
\sum=\left[\begin{array}{ll}
\sigma_{11} & \sigma_{12} \\
\sigma_{21} & \sigma_{22}
\end{array}\right]=\left[\begin{array}{cc}
\sigma_{1}^{2} & \rho \sigma_{1} \sigma_{2} \\
\rho \sigma_{1} \sigma_{2} & \sigma_{2}^{2}
\end{array}\right]
$$




$$
\rho=\frac{\sigma_{12}}{\sigma_{1} \sigma_{2}}
$$

é o coeficiente de correlação entre $X_{1}$ e $X_{2}, \sigma_{12}$ é a covariância entre $\mathrm{X}_{1}$ e $\mathrm{X}_{2}, \sigma_{i}$ é o desvio padrão da característica $i, \mathrm{i}=1,2$.

O determinante da matriz $\sum$, apresentada em (8) e (9) e definida em (10), pode ser calculado por $\left|\sum\right|=\sigma_{1}^{2} \sigma_{2}^{2}\left(1-\rho^{2}\right)$. A Figura 1 mostra uma distribuição normal bivariada.

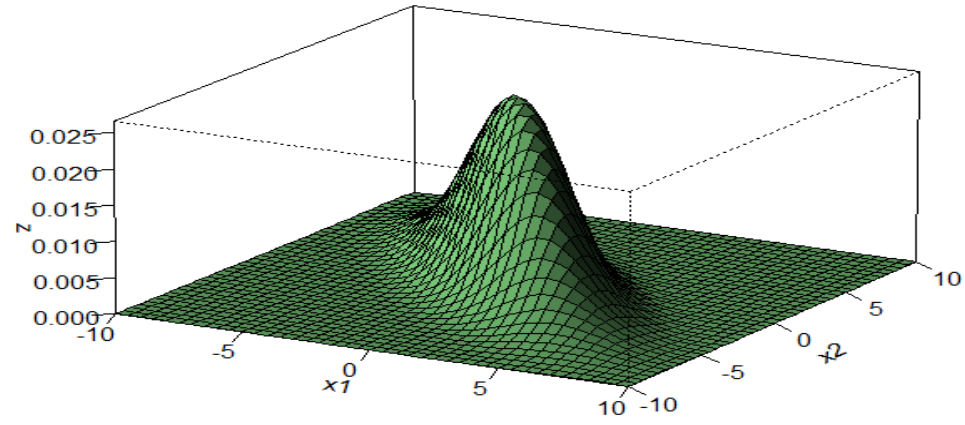

Figura 1 - Distribuição normal bivariada com $\rho=0,8, \mu^{\prime}=[0,0], \sigma_{1}^{2}=1$ e $\sigma_{2}^{2}=1$.

Para a verificação da suposição de normalidade multivariada foi utilizado o teste de Shapiro Wilk, em que as hipóteses são: H0: Os dados possuem distribuição normal multivariada e H1: Os dados não possuem distribuição normal multivariada (JAREK, 2013). Para a verificação da significância do coeficiente de correlação foram utilizadas as hipóteses H0 e H1, isto é, H0: $\rho=0$ (não existe correlação linear) e H1: $\rho \neq 0$ (existe correlação linear). Foi adotado para ambos os testes um nível de significância de $5 \%$.

Como as quantidades apresentadas em (8), (9) e (10), tais como o vetor de médias, as variâncias e covariâncias são desconhecidas, as mesmas são estimadas. A seguir, apresentou-se segundo Montgomery (2009) a estimação destas quantidades, com a adaptação para o tamanho de amostra variável.

Suponha uma amostra aleatória de uma distribuição normal multivariada no $k$-ésimo instante

$$
X_{1}, X_{2}, \ldots, X_{p}
$$

onde o $i$-ésimo componente do vetor amostral contém as observações $X_{i 1}, X_{i 2}, \ldots, X_{i n_{k}}$, com i $=1,2$, $\ldots, \mathrm{p}$ e $\mathrm{k}=1,2, \ldots, \mathrm{m}$. A cada instante é obtida cada uma das matrizes em (13)

$$
\left[\begin{array}{ccc}
X_{11} & \cdots & X_{p 1} \\
\vdots & \ddots & \vdots \\
X_{1 n_{1}} & \cdots & X_{p n_{1}}
\end{array}\right],\left[\begin{array}{ccc}
X_{11} & \cdots & X_{p 1} \\
\vdots & \ddots & \vdots \\
X_{1 n_{2}} & \cdots & X_{p n_{2}}
\end{array}\right], \cdots,\left[\begin{array}{ccc}
X_{11} & \cdots & X_{p 1} \\
\vdots & \ddots & \vdots \\
X_{1 n_{m}} & \cdots & X_{p n_{m}}
\end{array}\right],
$$

No $k$-ésimo instante, tem-se o vetor de médias, dado por $\bar{X}_{k}{ }^{\prime}=\left[\bar{X}_{1 k}, \ldots, \bar{X}_{p k}\right]^{\prime}$, com o $i$-ésimo componente obtido por

$$
\bar{X}_{i k}=\frac{1}{n_{k}} \sum_{j=1}^{n_{k}} X_{i j k}, \mathrm{i}=1,2, \ldots, \mathrm{p} \mathrm{e} \mathrm{k}=1,2, \ldots, \mathrm{m},
$$

onde $X_{i j k}$ é o valor da $i$-ésima característica, na j-ésima observação e no $k$-ésimo instante. A matriz de covariância amostral no $k$-ésimo instante é

$$
S_{k}=\left[\begin{array}{ccc}
S_{1 k}^{2} & \cdots & S_{1 p k} \\
\vdots & \ddots & \vdots \\
S_{p 1 k} & \cdots & S_{p k}^{2}
\end{array}\right],
$$

com as variâncias amostrais na diagonal principal da matriz $S_{\mathrm{k}}$ calculadas por

$$
S_{i k}^{2}=\frac{1}{n_{k}-1} \sum_{j=1}^{n_{k}}\left(X_{i j}-\bar{X}_{i}\right)^{2}
$$

e as covariâncias amostrais, calculadas

$$
S_{i i^{\prime} k}=\frac{1}{n_{k}-1} \sum_{j=1}^{n_{k}}\left(X_{i j}-\bar{X}_{i}\right)\left(X_{i^{\prime} j}-\bar{X}_{i^{\prime}}\right),
$$


para $i \neq i^{\prime}$. A literatura apresenta que a média dos $m$ instantes do vetor média amostral e da matriz de covariância amostral são estimadores não-viesados das quantidades populacionais correspondentes, isto é,

$$
E(\overline{\bar{X}})=\mu \text { e } E(\bar{S})=\sum \text {. }
$$

\subsection{Gráfico de controle multivariado para tamanho amostral variável}

O gráfico de controle multivariado (GCM) $\mathrm{T}^{2}$ de Hotelling é um gráfico análogo ao gráfico Xbarra de Shewhart univariado e é utilizado no monitoramento simultâneo de $p$ características da qualidade. Além disso, apresenta mais sensibilidade do que os gráficos univariados, permitindo ao operador detectar mais rapidamente os possíveis problemas existentes no processo e, com isso, corrigilos com mais agilidade. Cabe ressaltar que antes de iniciar uma análise de um processo é necessário avaliar se o mesmo contém características da qualidade independentes ou correlacionadas, pois um gráfico de controle inadequado poderá produzir alarmes falsos sobre o processo.

Esse gráfico foi desenvolvido por Hotelling (1947), o pioneiro na pesquisa sobre os gráficos de controle multivariados. Ele utilizou a abordagem multivariada de controle em dados contendo informações sobre localizações de bombardeios na Segunda Guerra Mundial. Hotelling apresenta a teoria considerando um tamanho de amostra $n$ fixo, ou seja, a cada instante tem-se o mesmo número de observações das $p$ características. Neste trabalho, ajusta-se a teoria proposta por Hotelling para $\mathrm{n}$ variável, uma vez que o estudo de caso considerado a seguir considera o tamanho amostral variável.

A estatística $\mathrm{T}^{2}$ de Hotelling para $n$ variável é definida da seguinte maneira

$$
T_{k}^{2}=n_{k}(\bar{X}-\overline{\bar{X}})^{\prime} \bar{S}^{-1}(\bar{X}-\overline{\bar{X}}),
$$

em que $\overline{\bar{X}}$ é a estimativa do vetor de médias de dimensão $p$ x 1 e $\bar{S}$ é a estimativa da matriz de variâncias e covariâncias do processo com dimensão $p \times p$.

$\mathrm{O}$ vetor $\overline{\overline{\mathrm{X}}}$ é calculado da seguinte maneira

$$
\overline{\bar{X}}=\left[\begin{array}{c}
\frac{1}{m} \sum_{k=1}^{m} \bar{X}_{1 k} \\
\vdots \\
\frac{1}{m} \sum_{k=1}^{m} \bar{X}_{p k}
\end{array}\right]=\left[\begin{array}{c}
\overline{\bar{X}}_{1} \\
\vdots \\
\overline{\bar{X}}_{p}
\end{array}\right]_{p x 1}
$$

em que, $\bar{X}_{i k}$ é a média da $i$-ésima característica, no $k$-ésimo instante, definida em (14). O vetor dado em (20) é obtido por meio da média das médias das amostras coletadas, ou seja, é a média de todos os $m$ valores obtidos em (14), de cada uma das características.

Já a estimativa de $\bar{S}$ é obtida por:

$$
\bar{S}=\left[\begin{array}{llll}
\frac{1}{m} \sum_{k=1}^{m} S_{(11) k} & \frac{1}{m} \sum_{k=1}^{m} S_{(12) k} & \cdots & \frac{1}{m} \sum_{k=1}^{m} S_{(1 p) k} \\
\frac{1}{m} \sum_{k=1}^{m} S_{(21) k} & \frac{1}{m} \sum_{k=1}^{m} S_{(22) k} & \cdots & \frac{1}{m} \sum_{k=1}^{m} S_{(2 p) k} \\
\vdots & \vdots & \ddots & \vdots \\
\frac{1}{m} \sum_{k=1}^{m} S_{(p 1) k} & \frac{1}{m} \sum_{k=1}^{m} S_{(p 2) k} & \cdots & \frac{1}{m} \sum_{k=1}^{m} S_{(p p) k}
\end{array}\right]=\left[\begin{array}{cccc}
\overline{S_{1}^{2}} & \bar{S}_{12} & \cdots & \bar{S}_{1 p} \\
& \bar{S}_{2}^{2} & \cdots & \bar{S}_{2 p} \\
& \ddots & \vdots \\
& & \bar{S}_{p}^{2}
\end{array}\right]_{p x p}
$$

em que, $S_{(i i) k}$ é a variância da $i$-ésima característica, no $k$-ésimo instante, definida em (16) e $S_{(i i) k}$ é a covariância entre a i-ésima e $i^{\prime}$-ésima característica, no k-ésimo instante, definida em (17). A matriz em (21) é obtida por meio da média das variâncias e covariâncias das amostras coletadas, ou seja, é a média de todos os $m$ valores obtidos em (16) e (17), das características estudadas. 
A expressão da estatística $\mathrm{T}^{2}$ em (19) é utilizada para construir os pontos do gráfico de controle multivariado $\mathrm{T}^{2}$ de Hotelling quando o tamanho da amostra é variável. Após calcular a estatística $\mathrm{T}^{2}$ é necessário calcular os limites de controle.

Alt (1985) observou que há duas fases distintas no uso de um gráfico de controle. A fase 1 é o uso dos gráficos para o estabelecimento do controle, ou seja, testar se o processo estava, ou não, sob controle quando os $m$ subgrupos preliminares foram extraídos e as estatísticas amostrais $\overline{\bar{X}}$ e $\bar{S}$, calculadas. $O$ objetivo na fase 1 é a obtenção de um conjunto de observações sob controle, de modo que os limites de controle possam ser estabelecidos para a fase 2, que é o monitoramento da produção futura. Isto é, às vezes, chamado análise retrospectiva.

Para realizar o cálculo do limite superior de controle do GCM $\mathrm{T}^{2}$ de Hotelling quando o tamanho de amostra é variável é utilizada a expressão (22). Cada ponto do gráfico terá um limite superior de controle específico dado por

$$
\begin{array}{rl}
L S C_{k}= & \frac{p(m-1)\left(n_{k}-1\right)}{m n_{k}-m-p+1} F_{\alpha, p,} m n_{k}-m-p+1 \\
\mathrm{e} & \mathrm{LIC}=0
\end{array}
$$

$\mathrm{Na}$ segunda fase de controle, os novos limites são estabelecidos apenas para monitorar as observações futuras, utilizando os limites de controle apresentados na equação (23). Em que cada ponto também terá um limite superior de controle específico dado por

$$
\begin{gathered}
L S C_{k}=\frac{p(m+1)\left(n_{k}-1\right)}{m n_{k}-m-p+1} F_{\alpha, p, m n_{k}-m-p+1} \\
\mathrm{e} \\
\text { LIC }=0
\end{gathered}
$$

\subsection{Decomposição da estatística $T^{2}$ para tamanho amostral variável}

Alencar, Lopes e Souza (2007) utilizaram uma abordagem para o diagnóstico de um sinal fora de controle, que é a decomposição da estatística $\mathrm{T}^{2}$ em componentes. A mesma foi proposta por Runger, Alt e Montgomery (1996). As componentes originadas pela decomposição refletem a contribuição de cada variável (ou característica de qualidade) individual. Se $T_{k}^{2}$ é o valor da estatística $T^{2}$ no $k$-ésimo instante e $T_{k(i)}^{2}$ é o valor da estatística para todas as variáveis do processo exceto a $i$ ésima característica da qualidade, então a expressão

$$
d_{k i}=T_{k}^{2}-T_{k(i)}^{2}, \mathrm{k}=1,2, \ldots, \mathrm{m}, \mathrm{i}=1,2, \ldots, \mathrm{p}
$$

é um indicador da contribuição relativa da $i$-ésima variável para a estatística global, no instante $k$. Quando um sinal fora de controle é gerado, é recomendado calcular os valores de $d_{k i}(\mathrm{i}=1,2, \ldots, \mathrm{p})$, apenas no instante $k$ que o ponto foi sinalizado e concentrar a atenção nas variáveis para os quais os $d_{k i}$ são maiores.

Para calcular o valor de $T_{k(i)}^{2}$ é necessário realizar o cálculo da matriz de variâncias e covariâncias a cada exclusão da i-ésima variável. Portanto, com o valor da estatística original $T_{k}^{2}$ e da $T_{k(i)}^{2}$ pode-se determinar o parâmetro $d_{k i}$ e avaliar qual característica da qualidade está afetando o processo no k-ésimo instante, que se mostrou acima do limite superior de controle definido em (22).

A decomposição da estatística $\mathrm{T}^{2}$ dá uma diretriz sobre quais variáveis mudaram no vetor de observações.

\subsection{Software livre $R$ versão 3.1.0}

O software utilizado para o desenvolvimento deste trabalho foi o $\mathrm{R}$ versão 3.1.0. O mesmo está disponível para diversos sistemas operacionais. O R possui diversos pacotes, ou bibliotecas, que englobam diversas ferramentas específicas para cada função pretendida. Neste trabalho foram utilizados os pacotes: $q c c$ versão 2.6 (Quality Control Charts) (SCRUCCA, 2015), para a geração dos gráficos univariados e o mvnormtest versão 0.1-9 (Normality test for multivariate variables) (JAREK, 2015), para a verificação da suposição de normalidade bivariada, no qual possui uma generalização do teste de Shapiro Wilk, para variáveis multivariadas. 


\section{Metodologia}

Essa seção contém o método adotado neste trabalho, que foi implementar a teoria apresentada na seção anterior no software livre R, com a estrutura do banco de dados reais de uma empresa que produz itens de segurança para automóveis que está localizada na região de São João del Rei - MG (ver Anexo 1).

\subsection{Estrutura dos dados}

Após acompanhar a linha de produção da empresa, foi verificado que era necessário realizar a análise nas hastes metálicas de $24 \mathrm{~mm}$ (aços utilizados para produção de itens de segurança automotivos), pois caso as mesmas não estivessem dentro dos limites de controle, isto acarretaria em peças defeituosas nas próximas etapas do processo. Portanto, comprometeria a durabilidade e resistência das mesmas, porque indicaria que os aços utilizados estariam sob condições desfavoráveis ao uso.

Para a melhoria da qualidade das hastes metálicas foi identificado que as características da qualidade têmpera e revenimento eram as variáveis que implicavam em seu resultado final. Estas variáveis são referentes a tratamentos térmicos (medidas de dureza). Os dados coletados têm uma escala de medida entre 75 e 81 HRC (dureza Rockwell na escala C) para têmpera e entre 72 e 78 HRC para revenimento.

$\mathrm{O}$ experimento era realizado em amostras selecionadas aleatoriamente da seguinte maneira: $\mathrm{O}$ primeiro processo realizado é o de têmpera. A haste selecionada é fixada na máquina e um indutor magnético realiza o aquecimento da mesma. Simultaneamente, uma ducha de água fria é lançada e ao final desta etapa, a informação sobre a dureza da haste é coletada e o processo é finalizado. Posteriormente, é realizado o processo de revenimento com o mesmo material, máquina e procedimento. Entretanto, com uma temperatura menor, pois é apenas para aliviar as tensões internas do material. Vale ressaltar que a máquina é programada para coletar as informações após o processo de têmpera e logo em seguida realizar o processo de revenimento.

Para investigar o processo, foram utilizadas informações dessas duas características da qualidade. Os dados coletados tinham o tamanho de amostra variável devido ao fluxo de produção da empresa que não era padrão, pois a mesma realiza o processo de acordo com a demanda de seus clientes que é variável. A coleta era realizada, em geral, de 2 em 2 horas, isto é, o ensaio demora em torno de 2 horas até ser concluído, coletando um valor para têmpera e um valor para revenimento a cada instante. No final de cada dia, o tamanho amostral oscilava entre 7 e 12 observações. Os dados utilizados neste trabalho são do período entre 1 a 31 de outubro de 2012, contabilizando um total de 31 amostras. A empresa estudada tem a sua produção contínua, inclusive em finais de semana e feriados (ver Anexo 1).

\subsection{Implementação computacional}

Para a implementação computacional, foi utilizado o software $\mathrm{R}$ versão 3.1.0. A seguir apresentou-se uma descrição dos programas disponíveis nos Anexos 2 e 3.

\subsubsection{Gráficos univariados Xbarra e S}

Para a geração dos gráficos univariados apresentados na Seção 2.2 (Xbarra e S), primeiro instalou-se o pacote $q c c$.

Posteriormente, entrou-se com todos os valores coletados da característica 1 (têmpera). Note que é necessário verificar qual foi o instante $k$ em que o tamanho amostral $\left(n_{k}\right)$ foi maior. Com isso, os demais instantes foram complementados com $N A$, para que todos instantes tivessem o mesmo tamanho. $\mathrm{O}$ mesmo foi feito para a característica da qualidade 2 (revenimento).

Por último, utilizou-se o comando qcc para gerar os gráficos Xbarra e S para cada uma das características. 


\subsubsection{Análise multivariada}

Nesta seção, foram apresentados os detalhes metodológicos das rotinas desenvolvidas para a construção do GCM T2 de Hotelling quando $n$ é variável (ver Anexo 3). O algoritmo computacional foi construído adaptando a teoria apresentada na Seção 2 com os dados disponíveis pela empresa (ver Anexo 1), obtidos conforme a descrição na Seção 3.1.

Os dados são referentes às $m=31$ amostras com tamanho variável, com $p=2$ características de qualidade, em que as características da qualidade foram escolhidas pela empresa, pois são as que mais afetam negativamente no produto final.

Para a geração do gráfico $\mathrm{T}^{2}$ de Hotelling no software $\mathrm{R}$ para $n$ variável foram seguidos os seguintes passos: primeiro foi necessário entrar com os dados coletados das 31 amostras de tamanho $n_{k}, \mathrm{k}=1, \ldots, 31$, das $p=2$ características da qualidade. Nesta situação, como $p=2, \mathrm{X}_{1}$ e $\mathrm{X}_{2}$ foram denominados por $\mathrm{X}_{\mathrm{k}}$ e $\mathrm{Y}_{\mathrm{k}}, \mathrm{k}=1,2, \ldots, 31$. Com isso, foram obtidos 2 vetores para cada instante. Por exemplo, observando o Anexo 3, no primeiro instante foram coletadas $n_{l}=8$ observações de cada característica, no instante $2, n_{2}=12$ observações, até o $31^{\circ}$ instante, onde foram coletadas $n_{31}=10$ dados. Em seguida, foi feito o teste de hipóteses para a correlação, através do comando cor.test e a verificação da suposição de normalidade bivariada através do comando mshapiro.test, que precisa da instalação do pacote mvnormtest, para ser executado. Após a realização destes testes, foi construída uma matriz contendo a média de cada instante da característica 1, de dimensão 31 linhas por 1 coluna (media1) e, outra matriz com a média de cada instante da característica 2, também de dimensão 31 x 1 (media2). Foi repetido o mesmo procedimento para as variâncias (variancia1, variancia2) e a covariância de cada instante entre as características da qualidade (covar). Posteriormente, calculou-se a média das médias (xbarbar1, xbarbar2) e criou-se uma matriz com esses resultados de dimensão $2 \mathrm{x}$ 1, calculou-se a média das variâncias (medvar1, medvar2) e a média das covariâncias (medcov).

Para criar a matriz de variâncias e covariâncias, primeiro criou-se uma matriz de zeros na dimensão desejada (2 linhas e 2 colunas). Mais especificamente, a primeira linha e primeira coluna dessa matriz recebeu o valor da média das variâncias da característica 1 da qualidade (medvar1); a primeira linha e segunda coluna e, a segunda linha e primeira coluna receberam o valor da média das covariâncias entre as duas características da qualidade (medcov) e, por último, a segunda coluna e segunda linha da matriz recebeu o valor da média das variâncias da segunda característica da qualidade (medvar2).

Em seguida, identificou-se o tamanho amostral, o número de características da qualidade avaliadas e o número de amostras. Como também, o vetor de média das amostras. Além disso, foi formulado um comando para o cálculo da estatística $\mathrm{T}^{2}$ de cada amostra com seu respectivo tamanho (ver fórmula (19)). Posteriormente, obteve-se o valor tabelado da distribuição F, utilizando um grau de confiança de $95 \%$. Com isso, foi possível colocar o comando adequado para o cálculo de cada limite superior de controle de cada amostra $m$ (ver fórmula (22)).

Com os resultados obtidos, foram utilizados os comandos plot e lines, para gerar o GCM T ${ }^{2}$ de Hotelling com tamanho da amostra variável. O comando points foi utilizado para sinalizar os pontos que ultrapassaram o limite superior de controle.

Por último, para os pontos sinalizados fora de controle no gráfico $\mathrm{T}^{2}$ de Hotelling, foi obtida a decomposição da estatística T ${ }^{2}\left(d_{k i}\right)$, conforme expressão em (24).

\section{Resultados e Discussões}

Com a teoria apresentada na Seção 2 e a metodologia ilustrada na Seção 3, apresenta-se nesta seção os resultados e discussões dos dados da empresa que produz itens de segurança para automóveis, que está localizada na região de São João del Rei - MG.

A empresa utilizava para monitorar o processo os gráficos de controle univariados Xbarra e $\mathrm{S}$ e os mesmos eram gerados no Excel. Para aprimorar o seu processo de produção, a organização permitiu introduzir novas técnicas e ferramentas de controle de processos, com intuito de garantir uma avaliação mais rigorosa da produção. Cabe ressaltar que foi possível trocar o uso do Excel pelo software livre R, que possibilitou a verificação das suposições de normalidade e correlação entre as características, a geração do gráfico multivariado e a decomposição da estatística $\mathrm{T}^{2}$ de Hotelling, sem nenhum custo adicional para a empresa. 


\subsection{Gráficos de controle univariados}

Para dar início à investigação do processo foi realizado o estudo utilizando os gráficos univariados Xbarra e S, gerados por meio do software livre R, versão 3.1.0 (ver Figuras 2, 3, 4 e 5). O algoritmo computacional utilizado está no Anexo 2.

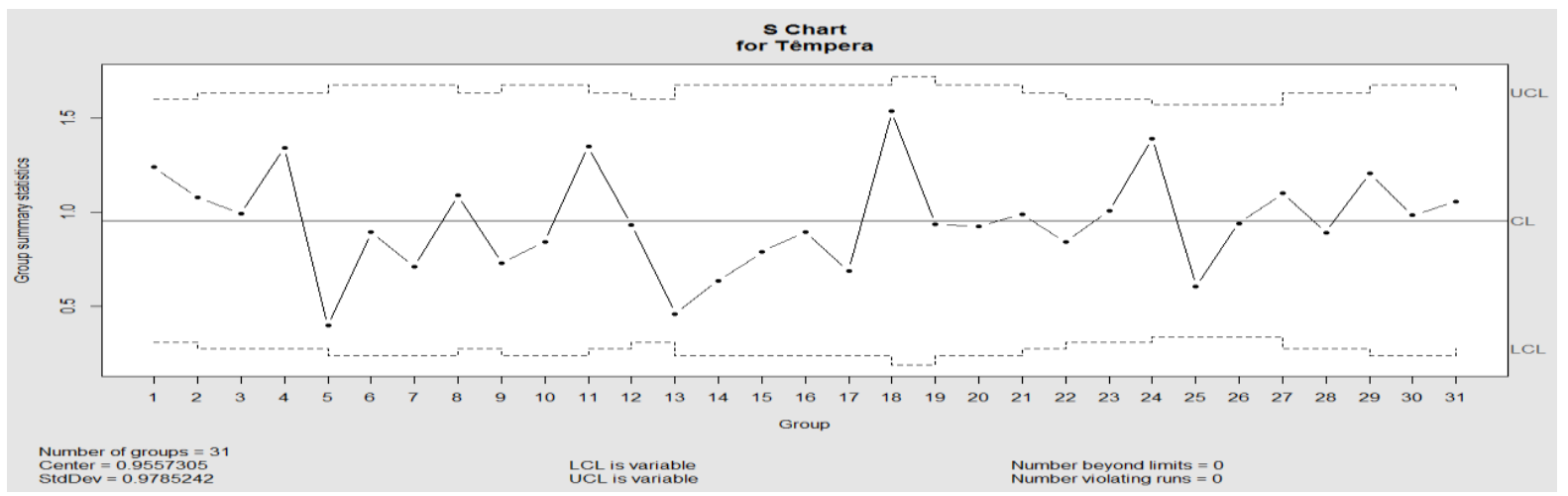

Figura 2 - Gráfico da variabilidade para a característica Têmpera.

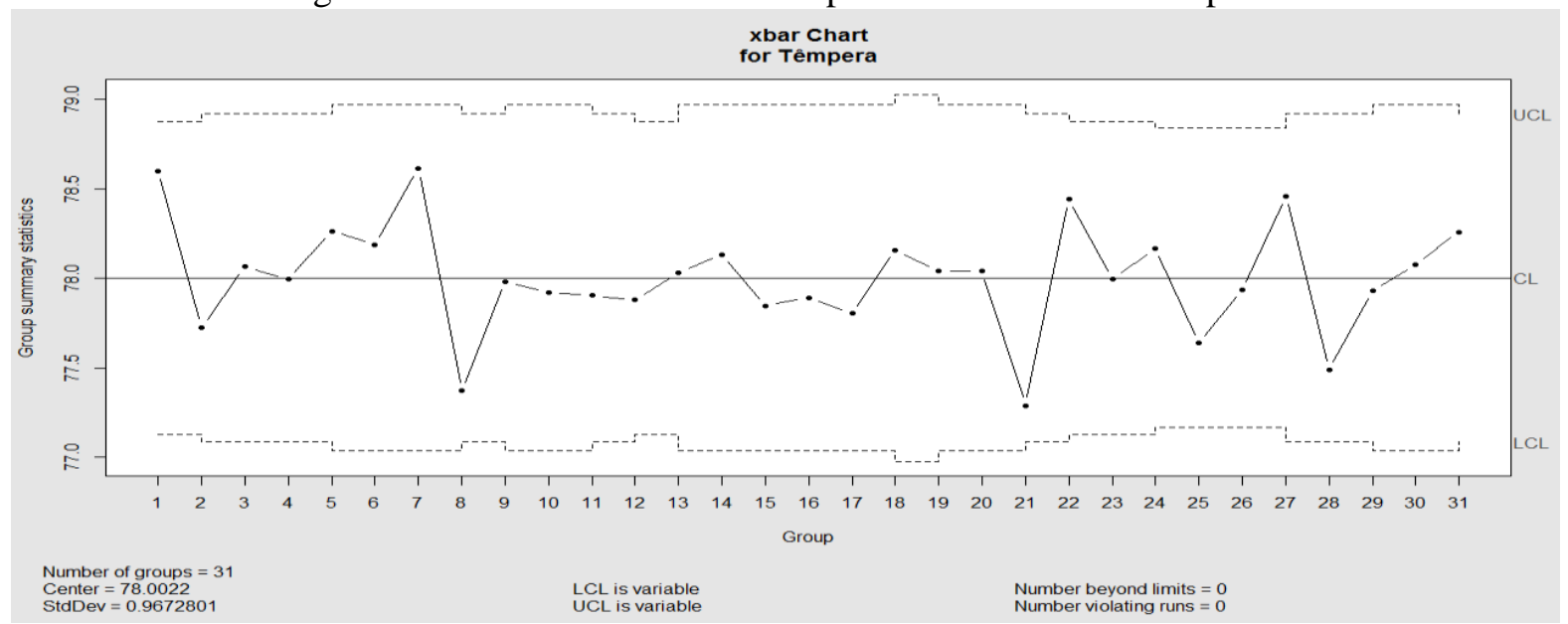

Figura 3 - Gráfico da média para a característica Têmpera.

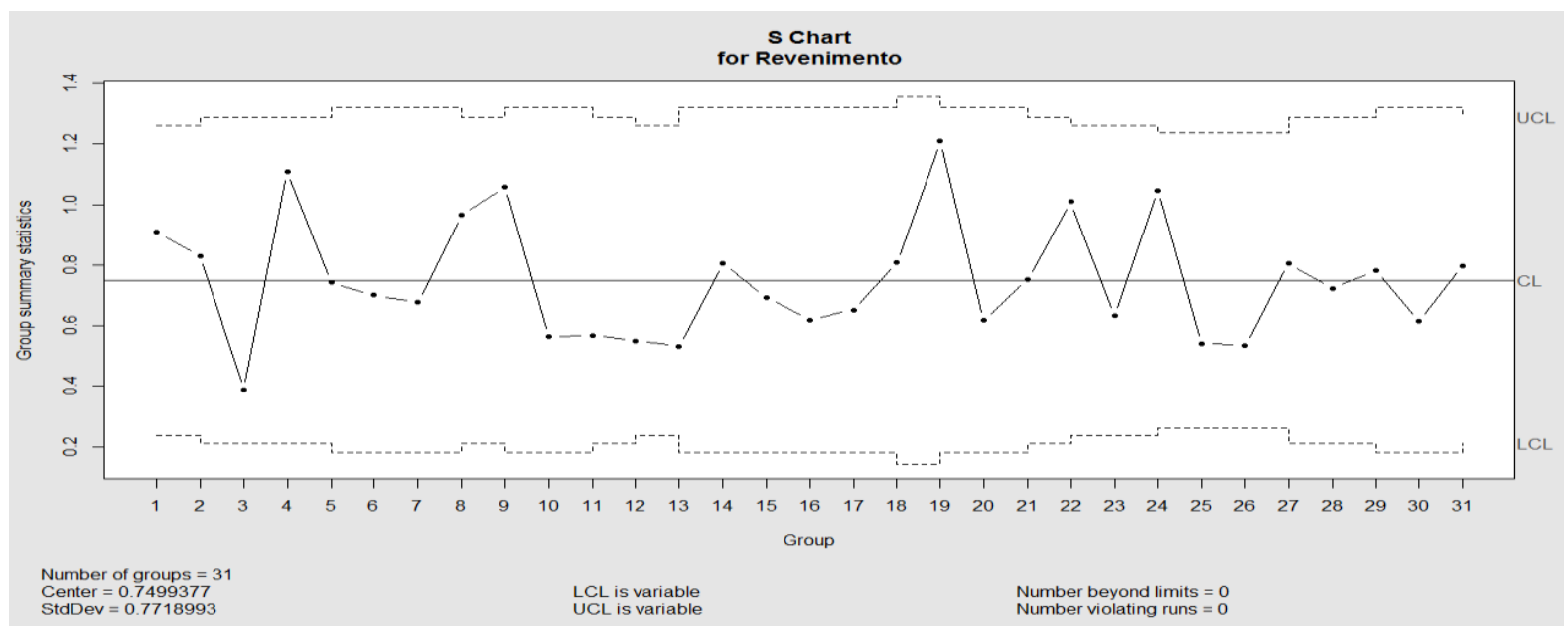

Figura 4 - Gráfico da variabilidade para a característica Revenimento. 


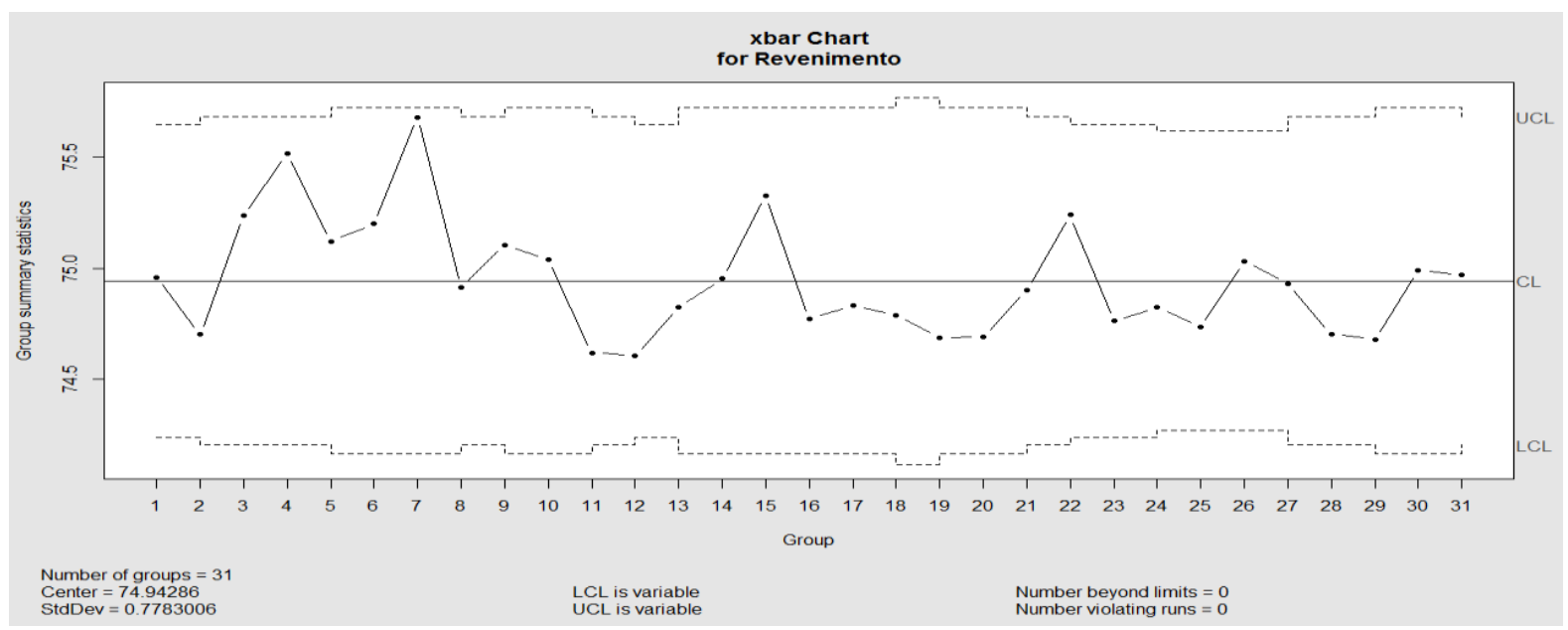

Figura 5 - Gráfico da média para a característica Revenimento.

Observando as Figuras 2, 3, 4 e 5 percebe-se que o processo encontra-se sob controle estatístico, isto é, as figuras indicam que não há causas especiais interferindo no processo, não havendo necessidade de realizar uma investigação do processo de produção da empresa.

\subsection{Análise multivariada}

Com o objetivo de apresentar a análise simultânea das duas características da qualidade, foi proposta a aplicação do GCM T2 de Hotelling. Para isso, foram realizados o teste de correlação e o teste de normalidade bivariada das características estudadas utilizando o software R, com um nível de significância de 5\% (ver Anexo 3). Os testes verificaram se realmente era viável a aplicação do GCM $\mathrm{T}^{2}$ de Hotelling, tendo como intuito, avaliar as suposições necessárias de correlação e normalidade dos dados.

Portanto, aplicando o teste da correlação foi verificado que há uma correlação linear positiva significativa entre as variáveis têmpera e revenimento (correlação $=0,43 ; \mathrm{t}=8,3$; graus de liberdade $=$ 302; p-valor $=4,219 \times 10^{-15}$ ). Além disso, a suposição de normalidade bivariada também foi comprovada, pois por meio do teste de Shapiro Wilk ( $\mathrm{W}=0,9973$ e $\mathrm{p}$-valor $=0,9)$.

Verificando que a correlação entre as características é significativa e a suposição de normalidade bivariada é garantida, a literatura recomenda que as características sejam avaliadas simultaneamente por meio do GCM T2 de Hotelling. O modelo computacional está no Anexo 3 e os detalhes metodológicos descritos na Seção 3.2.2. O gráfico gerado pelo software R para o processo em estudo é representado pela Figura 6. 


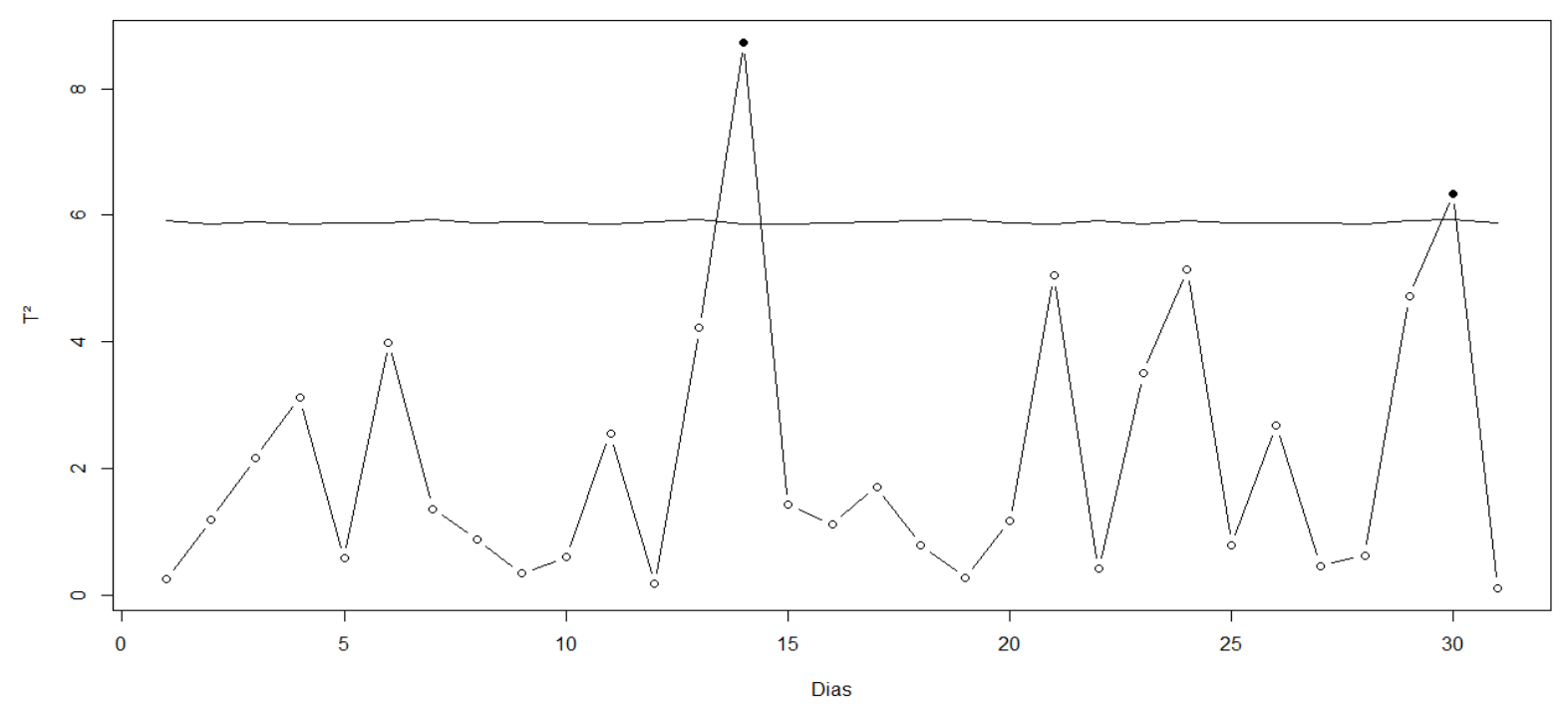

Figura 6-GCM T² de Hotelling para tamanho de amostra variável para as características Têmpera e Revenimento.

Observando a Figura 6, percebe-se que, quando as características são avaliadas simultaneamente, mostram que as amostras 14 e 30 saíram do limite superior de controle, o que indica que o processo não está sob controle estatístico. O próximo passo foi avaliar qual característica da qualidade estava impactando de forma negativa no processo, para que possa ser feito uma avaliação do mesmo. Realizando a decomposição da estatística $\mathrm{T}^{2}$ (ver Anexo 3) para os pontos que ultrapassaram o limite superior de controle, foi detectado que, com relação à amostra 14 a característica revenimento afetou o processo, pois $\mathrm{d}_{14,2}$ apresentou um valor superior a $\mathrm{d}_{14,1}$. Como também, para a amostra 30 , a característica revenimento é a que estava impactando no processo de forma negativa, porque o valor de $\mathrm{d}_{30,2}$ foi superior ao de $\mathrm{d}_{30,1}$. Com isso, foi possível proporcionar à empresa um direcionamento de quais características precisavam ser investigadas nos seus respectivos instantes.

Com relação à amostra 14, no dia 14/10/2012, foi possível pontuar que o operador da máquina que realizava este processo e responsável pelos ensaios, não realizou o processo de Revenimento de forma adequada, pois as medidas coletadas deveriam apresentar resultados com valores maiores, indicando maior resistência de dureza da peça. Desta forma, uma possível justificativa foi o lançamento do jato de água em menor quantidade, e/ou em tempo não satisfatório do lançamento do jato de água para que a peça contenha melhor desempenho. Essa mesma situação ocorreu para a amostra 30, no dia 30/10/2012.

\section{Considerações finais}

O objetivo desse estudo foi apresentar a teoria composta para a geração do gráfico multivariado proposto por Hotelling, quando o tamanho de amostra é variável, fazer a implementação computacional desta teoria num software livre e realizar um estudo de caso numa empresa da região de São João del Rei-MG.

O estudo de caso possibilitou mostrar a importância do uso do gráfico multivariado, pois a empresa utilizava somente as cartas univariadas e, os resultados obtidos com esses gráficos não eram eficazes para o monitoramento da sua produção, uma vez que a empresa avaliava mais que uma característica da qualidade simultaneamente e as mesmas eram correlacionadas. Em particular, para o período analisado na empresa, os resultados mostraram que com o uso das cartas univariadas não era detectado nenhuma causa especial no processo e, em contrapartida, com o gráfico $\mathrm{T}^{2}$ de Hotelling foi possível detectar 2 pontos fora de controle, sinalizados devido à característica revenimento e, além disso, detectar que tipo de problema estava ocorrendo com esta característica da qualidade. Além disso, a empresa utilizava um software que não era livre, isto é, tinha um custo adicional para obter a licença do mesmo e, este trabalho, proporcionou uma alternativa de custo zero.

Portanto, esse trabalho contribuiu fornecendo a rotina computacional do gráfico multivariado e de sua análise num software estatístico gratuito e os dados da empresa mostraram que os gráficos 
univariados não eram eficazes para o controle daquele processo. O programa disponível nos Anexos 2 e 3 possibilitará outras empresas no monitoramento multivariado, quando o tamanho amostral é fixo ou variável. $\mathrm{O}$ algoritmo computacional desenvolvido é didático e permite que seja desenvolvido o controle e a garantia da qualidade de produtos/serviços, sem custos adicionais com a compra de softwares. Além disso, a rotina computacional para a análise multivariada em um software livre é escassa e a mesma auxilia na melhoria contínua da qualidade dos produtos, produzindo melhores equipamentos no mercado e, consequentemente, mantém a competitividade e o segmento da empresa.

\section{Referências}

ALENCAR, J. R. B.; LOPES, C. E.; SOUZA Jr, M. B. Controle Estatístico de Processo Multivariado: aplicação ao monitoramento da produção de comprimidos de captopril. Revista Brasileira de Farmacologia, v. 88, n. 4, p. 200-205, 2007.

ALT, F. B. Multivariate Quality Control. In Encyclopedia of Statistical Sciences, v. 6, edited by N. L. Johnson and S. Kotz, Wiley, New York.

COSTA, A. F. B; EPPRECHT, E. K.; CARPINETTI, L. C. R. Controle Estatístico de Qualidade. 2 ed. São Paulo: Atlas, 2012.334p.

FOLlador, F. A. C. Controle Estatístico de Processo Aplicado À Qualidade de Águas Superficiais. 2010. 132f. Tese (Doutorado em Engenharia Agrícola) - Centro de Ciências Exatas e Tecnológicas, Universidade Estadual do Oeste do Paraná, Cascavel, 2010.

HOTELling, H. Multivariate Quality Control - illustrated by the air testing of sample bombsights. Techniques of Statistical Analysis. McGraw Hill, New York, 1947.

JAREK, S. Normality test for multivariate variables. Package 'mvnormtest'. CRAN, 2015. Disponível em: 〈http://cran.r-project.org/web/packages/mvnormtest/mvnormtest.pdf〉. Acesso em 25 de março de 2015.

LIMA, M. B. F.; SANTOS, R. L. S.; SOUZA, E. L.; SILVA, L. B. Aplicações do Controle Estatístico Multivariado da Qualidade: Controlando Garrafeiras Plásticas numa Empresa Industrial. Cadernos do IME - Série Estatística, v. 32, p. 01 - 14, 2012.

MELO, G. M. Decomposição da Estatística Teste do Gráfico de Controle Multivariado $\mathbf{T}^{\mathbf{2}}$ de Hotelling para Monitoramento e Avaliação do Controle de Processos de Agências Bancárias. 2008. 90 f. Dissertação (Mestrado em Estatística) - Instituto de Ciências Exatas e Naturais, Universidade Federal do Pará, Belém. 2008.

MONTGOMERY, D. C. Introduction to Statistical Quality Control. 6 ed. United State of America: John Wiley \& Sons, Inc., 2009, 734p.

OYEYEMI, G. M. Principal Component Chart For Multivariate Statistical Process Control. The Online Journal of Science and Technology, v.1, n. 2, p.22 - 31, Abril, 2011.

R Development Core Team. R: A language and environment for statistical computing. Vienna: $R$ Foundation for Statistical Computing, 2014. Disponível em: $<$ http://www.r-project.org/ >. Acesso em: 20 de maio de 2014.

ROJAS, N.; La, O M.; ARECE, J.; CARRION, M.; PEREZ, K.; MARTIN, C. S.; VALERIANO, P.; RAMIREZ, W. Identificación y caracterización de especies de Haemonchus en caprinos del valle del Cauto en Granma. Revista Electrónica de Veterinaria, v. 13, n. 1, 2012. 
RUNGER, G. C.; ALT, F. B.; MONTGOMERY, D. C. Contributors to a Multivariate Statistical Process Control Signal. Communications in Statistics - Theory and Methods, v. 25, n. 10, 1996.

SAMOHYL, R. W. Controle estatístico de qualidade. Rio de Janeiro: Elsevier, 2009.

SCRUCCA, L. Quality Control Charts. Package 'qce'. CRAN, 2015. Disponível em: < http://cran.rproject.org/web/packages/qcc/qcc.pdf>. Acesso em 25 de março de 2015.

SINGH, Y.; KUMAR, M. Application of Statistical Methods to Analyze Groundwater Quality. Journal of Earth Sciences and Geotechnical Engineering, v. 1, n. 1, p. 1-7, 2011.

\section{Anexos}

Anexo 1 - Dados sobre as características da qualidade têmpera e revenimento no mês de outubro.

\begin{tabular}{|c|c|c|c|c|c|c|c|c|}
\hline Data & Temp. * & Reven. ** & Data & Temp. & Reven. & Data & Temp. & Reven. \\
\hline $01 / 10 / 2012$ & 76,8 & 74,29 & $11 / 10 / 2012$ & 79,02 & 75,2 & $21 / 10 / 2012$ & 76,99 & 74,33 \\
\hline 01/10/2012 & 76,41 & 73,43 & $11 / 10 / 2012$ & 80,36 & 76,37 & $21 / 10 / 2012$ & 77,68 & 73,45 \\
\hline 01/10/2012 & 79,8 & 75,14 & $11 / 10 / 2012$ & 80,13 & 76,1 & $21 / 10 / 2012$ & 78,72 & 74,98 \\
\hline 01/10/2012 & 78,12 & 76,39 & $11 / 10 / 2012$ & 77,98 & 74,21 & $21 / 10 / 2012$ & 76,41 & 75,23 \\
\hline $01 / 10 / 2012$ & 77,92 & 75,3 & $11 / 10 / 2012$ & 76,92 & 75,16 & $22 / 10 / 2012$ & 78,65 & 75,87 \\
\hline $01 / 10 / 2012$ & 78,72 & 75,66 & $11 / 10 / 2012$ & 76,01 & 73,87 & $22 / 10 / 2012$ & 77,4 & 73,99 \\
\hline 01/10/2012 & 78,95 & 75,52 & $11 / 10 / 2012$ & 78,42 & 74,71 & $22 / 10 / 2012$ & 77,7 & 76,48 \\
\hline 01/10/2012 & 77,81 & 74,81 & $11 / 10 / 2012$ & 77,23 & 74,47 & $22 / 10 / 2012$ & 77,45 & 73,53 \\
\hline 02/10/2012 & 78,64 & 75,08 & $11 / 10 / 2012$ & 78,6 & 76,4 & $22 / 10 / 2012$ & 77,71 & 74,48 \\
\hline 02/10/2012 & 76,99 & 73,45 & $11 / 10 / 2012$ & 76,94 & 75,63 & $22 / 10 / 2012$ & 77,55 & 75,19 \\
\hline $02 / 10 / 2012$ & 78,49 & 75,59 & $12 / 10 / 2012$ & 78,21 & 74,63 & $22 / 10 / 2012$ & 76,06 & 74,19 \\
\hline $02 / 10 / 2012$ & 75,98 & 74,71 & $12 / 10 / 2012$ & 78,84 & 76,08 & $22 / 10 / 2012$ & 79,77 & 74,74 \\
\hline $02 / 10 / 2012$ & 77,98 & 74,65 & $12 / 10 / 2012$ & 78,22 & 75,63 & $23 / 10 / 2012$ & 76,89 & 75,36 \\
\hline 02/10/2012 & 80,1 & 76,36 & $12 / 10 / 2012$ & 77,47 & 74,77 & $23 / 10 / 2012$ & 79,79 & 74,79 \\
\hline $02 / 10 / 2012$ & 76,91 & 72,46 & $12 / 10 / 2012$ & 77,58 & 74,35 & $23 / 10 / 2012$ & 76,65 & 73,65 \\
\hline $02 / 10 / 2012$ & 77,97 & 74,53 & $12 / 10 / 2012$ & 78,48 & 74,93 & $23 / 10 / 2012$ & 76,68 & 74,11 \\
\hline $02 / 10 / 2012$ & 76,47 & 75,11 & $12 / 10 / 2012$ & 77,55 & 74,12 & $23 / 10 / 2012$ & 75,23 & 73,77 \\
\hline $02 / 10 / 2012$ & 77,5 & 74,94 & $12 / 10 / 2012$ & 77,98 & 74,39 & $23 / 10 / 2012$ & 79,15 & 76,75 \\
\hline $02 / 10 / 2012$ & 77,63 & 74,89 & $13 / 10 / 2012$ & 78,55 & 76,38 & $23 / 10 / 2012$ & 77,34 & 74,2 \\
\hline $02 / 10 / 2012$ & 78,37 & 74,88 & $13 / 10 / 2012$ & 77,16 & 75 & $23 / 10 / 2012$ & 76,7 & 73,72 \\
\hline 03/10/2012 & 77,75 & 74,24 & $13 / 10 / 2012$ & 77,08 & 74,7 & $23 / 10 / 2012$ & 77,31 & 73,93 \\
\hline 03/10/2012 & 77,71 & 74,82 & $13 / 10 / 2012$ & 78,5 & 75,88 & $23 / 10 / 2012$ & 78,81 & 76 \\
\hline 03/10/2012 & 78,81 & 75,31 & $13 / 10 / 2012$ & 77,54 & 75,34 & $23 / 10 / 2012$ & 78,89 & 74,99 \\
\hline 03/10/2012 & 77,13 & 73,62 & $13 / 10 / 2012$ & 78,88 & 75,55 & $23 / 10 / 2012$ & 76,17 & 75,46 \\
\hline 03/10/2012 & 77,57 & 74,66 & $13 / 10 / 2012$ & 78,97 & 75,64 & $24 / 10 / 2012$ & 77,48 & 74,21 \\
\hline 03/10/2012 & 76,71 & 73,96 & $14 / 10 / 2012$ & 78,22 & 75,6 & $24 / 10 / 2012$ & 76,71 & 74,44 \\
\hline 03/10/2012 & 78,75 & 75,22 & $14 / 10 / 2012$ & 77,72 & 74,98 & $24 / 10 / 2012$ & 77,35 & 75,13 \\
\hline 03/10/2012 & 78,22 & 74,48 & $14 / 10 / 2012$ & 80,33 & 77,59 & $24 / 10 / 2012$ & 78,36 & 74,81 \\
\hline 03/10/2012 & 78,51 & 74,86 & $14 / 10 / 2012$ & 77,9 & 76,45 & $24 / 10 / 2012$ & 76,17 & 74,72 \\
\hline $04 / 10 / 2012$ & 77,25 & 75,88 & $14 / 10 / 2012$ & 79,03 & 75,77 & $24 / 10 / 2012$ & 77,43 & 75,58 \\
\hline 04/10/2012 & 78,98 & 75,35 & $14 / 10 / 2012$ & 78,97 & 76,09 & $24 / 10 / 2012$ & 77,05 & 75,4 \\
\hline $04 / 10 / 2012$ & 75,82 & 75,38 & $14 / 10 / 2012$ & 79,24 & 75,21 & $24 / 10 / 2012$ & 77,55 & 74,89 \\
\hline $04 / 10 / 2012$ & 78,71 & 75,9 & $14 / 10 / 2012$ & 78,59 & 76,38 & $25 / 10 / 2012$ & 79,04 & 75,13 \\
\hline
\end{tabular}




\begin{tabular}{|c|c|c|c|c|c|c|c|c|}
\hline 04/10/2012 & 76,93 & 74,37 & $14 / 10 / 2012$ & 78,19 & 74,55 & $25 / 10 / 2012$ & 75,63 & 74,24 \\
\hline 04/10/2012 & 78,73 & 74,84 & $14 / 10 / 2012$ & 79,52 & 74,77 & $25 / 10 / 2012$ & 78,83 & 74,81 \\
\hline 04/10/2012 & 77,23 & 74,69 & $14 / 10 / 2012$ & 77,85 & 74,13 & $25 / 10 / 2012$ & 77,4 & 75,9 \\
\hline $04 / 10 / 2012$ & 78,38 & 75,04 & $14 / 10 / 2012$ & 78,05 & 74,58 & $25 / 10 / 2012$ & 78,12 & 75,38 \\
\hline $04 / 10 / 2012$ & 78,96 & 76,09 & $15 / 10 / 2012$ & 78,67 & 75,03 & $25 / 10 / 2012$ & 77,77 & 74,68 \\
\hline 04/10/2012 & 77,71 & 76,12 & $15 / 10 / 2012$ & 76,2 & 74,68 & $25 / 10 / 2012$ & 78,21 & 75,13 \\
\hline 04/10/2012 & 78,73 & 75,47 & $15 / 10 / 2012$ & 77,72 & 73,91 & $25 / 10 / 2012$ & 78,15 & 74,48 \\
\hline 04/10/2012 & 78,39 & 74,28 & $15 / 10 / 2012$ & 78,51 & 75,35 & $25 / 10 / 2012$ & 77,02 & 74,75 \\
\hline 05/10/2012 & 78,66 & 75,48 & $15 / 10 / 2012$ & 76,58 & 73,85 & $25 / 10 / 2012$ & 78,38 & 76,1 \\
\hline 05/10/2012 & 77,77 & 73,58 & $15 / 10 / 2012$ & 78,14 & 74,81 & $26 / 10 / 2012$ & 79,22 & 74,7 \\
\hline 05/10/2012 & 79,57 & 75,33 & $15 / 10 / 2012$ & 77,81 & 74,72 & $26 / 10 / 2012$ & 77,69 & 74,82 \\
\hline 05/10/2012 & 76,93 & 74,24 & $15 / 10 / 2012$ & 77,81 & 74,83 & $26 / 10 / 2012$ & 77,4 & 73,93 \\
\hline 05/10/2012 & 79,72 & 76,05 & $15 / 10 / 2012$ & 78,35 & 75,77 & $26 / 10 / 2012$ & 77,36 & 74,59 \\
\hline 05/10/2012 & 76,21 & 73,94 & $15 / 10 / 2012$ & 77,99 & 74,33 & $26 / 10 / 2012$ & 78,17 & 75,07 \\
\hline 05/10/2012 & 76,63 & 74,19 & $15 / 10 / 2012$ & 78,43 & 74,46 & $26 / 10 / 2012$ & 79,07 & 75,02 \\
\hline 05/10/2012 & 78,03 & 74,99 & $15 / 10 / 2012$ & 79,14 & 74,48 & $26 / 10 / 2012$ & 78,07 & 74,24 \\
\hline 05/10/2012 & 77,5 & 74,72 & $16 / 10 / 2012$ & 77,59 & 75,11 & $26 / 10 / 2012$ & 79,59 & 74,91 \\
\hline 05/10/2012 & 78,08 & 76,23 & $16 / 10 / 2012$ & 78,32 & 74,9 & $26 / 10 / 2012$ & 78,6 & 75,88 \\
\hline 05/10/2012 & 76,84 & 73,84 & $16 / 10 / 2012$ & 78,14 & 75,76 & $26 / 10 / 2012$ & 77,31 & 74,56 \\
\hline 06/10/2012 & 78,46 & 75,59 & $16 / 10 / 2012$ & 77,81 & 74,63 & $26 / 10 / 2012$ & 80,17 & 76,53 \\
\hline 06/10/2012 & 79,07 & 76,26 & $16 / 10 / 2012$ & 77,22 & 75,53 & $27 / 10 / 2012$ & 78,72 & 75,69 \\
\hline 06/10/2012 & 80,22 & 75,97 & $16 / 10 / 2012$ & 77,34 & 74,94 & $27 / 10 / 2012$ & 79,75 & 75,45 \\
\hline 06/10/2012 & 77 & 74,57 & $16 / 10 / 2012$ & 77,47 & 74 & $27 / 10 / 2012$ & 78,73 & 74,4 \\
\hline 06/10/2012 & 78,94 & 75,91 & $16 / 10 / 2012$ & 76,96 & 75,66 & $27 / 10 / 2012$ & 77,72 & 75,21 \\
\hline 06/10/2012 & 77,69 & 74,1 & $16 / 10 / 2012$ & 78,41 & 74,88 & $27 / 10 / 2012$ & 78,16 & 75,93 \\
\hline 06/10/2012 & 78,51 & 75,65 & $16 / 10 / 2012$ & 78,46 & 74,96 & $27 / 10 / 2012$ & 76,27 & 74,1 \\
\hline 06/10/2012 & 78,17 & 74,97 & $17 / 10 / 2012$ & 79,84 & 74,25 & $27 / 10 / 2012$ & 78,13 & 74,82 \\
\hline 06/10/2012 & 77,56 & 75,37 & $17 / 10 / 2012$ & 78,99 & 75,02 & $27 / 10 / 2012$ & 78,53 & 75,59 \\
\hline 06/10/2012 & 76,91 & 75,21 & $17 / 10 / 2012$ & 78,54 & 76,53 & $27 / 10 / 2012$ & 77,99 & 75,14 \\
\hline 06/10/2012 & 78,27 & 75,68 & $17 / 10 / 2012$ & 78,21 & 75,14 & $27 / 10 / 2012$ & 77,95 & 73,5 \\
\hline 07/10/2012 & 77,24 & 74,89 & $17 / 10 / 2012$ & 78,64 & 74,76 & $28 / 10 / 2012$ & 79,11 & 75,52 \\
\hline 07/10/2012 & 77,91 & 74,23 & $17 / 10 / 2012$ & 76,22 & 74,67 & $28 / 10 / 2012$ & 77,87 & 74,35 \\
\hline 07/10/2012 & 78,09 & 74,97 & $17 / 10 / 2012$ & 78,62 & 74,91 & $28 / 10 / 2012$ & 77,98 & 75,08 \\
\hline 07/10/2012 & 77,54 & 74,74 & $17 / 10 / 2012$ & 78,54 & 74,83 & $28 / 10 / 2012$ & 77,1 & 74,25 \\
\hline 07/10/2012 & 78,05 & 75,84 & $17 / 10 / 2012$ & 78,08 & 75,31 & $28 / 10 / 2012$ & 78,2 & 75,44 \\
\hline 07/10/2012 & 77,36 & 74,49 & $18 / 10 / 2012$ & 77,81 & 75,42 & $28 / 10 / 2012$ & 78,25 & 75,68 \\
\hline 07/10/2012 & 76,77 & 74,1 & $18 / 10 / 2012$ & 78,55 & 75,22 & $28 / 10 / 2012$ & 78,32 & 74,97 \\
\hline 08/10/2012 & 79,06 & 74,04 & $18 / 10 / 2012$ & 76,99 & 74,48 & $28 / 10 / 2012$ & 78,18 & 72,82 \\
\hline 08/10/2012 & 75,97 & 73,36 & $18 / 10 / 2012$ & 77,96 & 75,15 & $28 / 10 / 2012$ & 79,21 & 75,84 \\
\hline 08/10/2012 & 78,19 & 75,55 & $18 / 10 / 2012$ & 78,96 & 75,7 & $28 / 10 / 2012$ & 80,2 & 75,49 \\
\hline 08/10/2012 & 79,25 & 74,9 & $18 / 10 / 2012$ & 77,99 & 75,43 & $28 / 10 / 2012$ & 77,08 & 74,77 \\
\hline 08/10/2012 & 78,08 & 75,38 & $18 / 10 / 2012$ & 77,43 & 74 & $28 / 10 / 2012$ & 76,02 & 74,06 \\
\hline 08/10/2012 & 79,08 & 74,71 & $18 / 10 / 2012$ & 78,12 & 75,77 & $29 / 10 / 2012$ & 76,53 & 73,47 \\
\hline 08/10/2012 & 75,6 & 75,26 & $19 / 10 / 2012$ & 77,21 & 74,25 & $29 / 10 / 2012$ & 78,08 & 75,42 \\
\hline 08/10/2012 & 79,31 & 74,62 & $19 / 10 / 2012$ & 78,9 & 73,98 & $29 / 10 / 2012$ & 78,75 & 73,31 \\
\hline 08/10/2012 & 77,7 & 74,14 & $19 / 10 / 2012$ & 78,96 & 74,84 & $29 / 10 / 2012$ & 78,72 & 74,84 \\
\hline
\end{tabular}




\begin{tabular}{rcccccccc}
\hline $08 / 10 / 2012$ & 78,15 & 75,53 & $19 / 10 / 2012$ & 76,86 & 75,34 & $29 / 10 / 2012$ & 78,37 & 74,32 \\
$09 / 10 / 2012$ & 78,21 & 75,72 & $19 / 10 / 2012$ & 77,91 & 76,43 & $29 / 10 / 2012$ & 79,18 & 74,52 \\
$09 / 10 / 2012$ & 78,23 & 74,48 & $19 / 10 / 2012$ & 78,47 & 74,33 & $29 / 10 / 2012$ & 79,38 & 75,7 \\
$09 / 10 / 2012$ & 79,84 & 76,05 & $19 / 10 / 2012$ & 78,34 & 74,81 & $29 / 10 / 2012$ & 77,14 & 74,69 \\
$09 / 10 / 2012$ & 78,75 & 75,71 & $20 / 10 / 2012$ & 78,56 & 74,77 & $30 / 10 / 2012$ & 76,76 & 73,58 \\
$09 / 10 / 2012$ & 77,52 & 76,68 & $20 / 10 / 2012$ & 77,55 & 74,54 & $30 / 10 / 2012$ & 77,12 & 73,9 \\
$09 / 10 / 2012$ & 76,55 & 73,62 & $20 / 10 / 2012$ & 78,3 & 75,89 & $30 / 10 / 2012$ & 77,33 & 74,39 \\
$09 / 10 / 2012$ & 76,66 & 74,9 & $20 / 10 / 2012$ & 77,66 & 76,07 & $30 / 10 / 2012$ & 77,33 & 73,81 \\
$09 / 10 / 2012$ & 79,68 & 73,93 & $20 / 10 / 2012$ & 79,16 & 74,59 & $30 / 10 / 2012$ & 77,58 & 75,75 \\
$09 / 10 / 2012$ & 77,3 & 74,63 & $20 / 10 / 2012$ & 78,44 & 76,01 & $30 / 10 / 2012$ & 77,96 & 74,04 \\
$10 / 10 / 2012$ & 78,02 & 75,9 & $20 / 10 / 2012$ & 79,59 & 74,71 & $30 / 10 / 2012$ & 77,6 & 74,24 \\
$10 / 10 / 2012$ & 77,13 & 74,19 & $20 / 10 / 2012$ & 78,7 & 74,92 & $31 / 10 / 2012$ & 78,71 & 74,6 \\
$10 / 10 / 2012$ & 76,44 & 74,12 & $20 / 10 / 2012$ & 76,69 & 74,26 & $31 / 10 / 2012$ & 77,08 & 74,35 \\
$10 / 10 / 2012$ & 79,8 & 75,81 & $20 / 10 / 2012$ & 77,38 & 75,64 & $31 / 10 / 2012$ & 77,58 & 75,3 \\
$10 / 10 / 2012$ & 78,12 & 73,78 & $20 / 10 / 2012$ & 78,81 & 75,1 & $31 / 10 / 2012$ & 79,29 & 74,68 \\
$10 / 10 / 2012$ & 78,13 & 75,24 & $21 / 10 / 2012$ & 80,19 & 73,91 & $31 / 10 / 2012$ & 77,79 & 76,03 \\
$10 / 10 / 2012$ & 79,24 & 76,05 & $21 / 10 / 2012$ & 77,3 & 74,76 & $31 / 10 / 2012$ & 77,69 & 75,51 \\
$10 / 10 / 2012$ & 77,51 & 75,25 & $21 / 10 / 2012$ & 76,57 & 74,7 & $31 / 10 / 2012$ & 78,54 & 75,06 \\
$10 / 10 / 2012$ & 79,83 & 76,6 & $21 / 10 / 2012$ & 80,58 & 74,3 & $31 / 10 / 2012$ & 77,26 & 74,06 \\
$10 / 10 / 2012$ & 76,84 & 74,65 & $21 / 10 / 2012$ & 77,64 & 74,74 & $31 / 10 / 2012$ & 76,42 & 74,21 \\
$10 / 10 / 2012$ & 78,07 & 74,61 & $21 / 10 / 2012$ & 77,09 & 74,22 & $31 / 10 / 2012$ & 79,19 & 74,76 \\
$11 / 10 / 2012$ & 77,33 & 75,05 & $21 / 10 / 2012$ & 77,93 & 74,85 & & & \\
$11 / 10 / 2012$ & 78,29 & 76,16 & $21 / 10 / 2012$ & 79,13 & 74,5 & & & \\
\hline$*$ Temp $:$ Têmpera $($ escala de $75-81) H R C$ & $* * 2$ & & & & \\
\hline
\end{tabular}

*Temp.: Têmpera (escala de 75-81) HRC **Reven.: Revenimento (escala 72-78) HRC

Fonte: Dados de uma empresa da região de São João del Rei-MG, que produz itens de segurança de automóveis.

Anexo 2 - Algoritmo computacional do software R para gerar os gráficos Xbarra e S com tamanho de amostra variável.

install.packages("qcc")

require(qcc)

Têmpera=matrix $(\mathrm{c}(76.8,76.41,79.8,78.12,77.92,78.72,78.95,77.81, N A, N A, N A, N A, 78.64,76.99,78.49$, 75.98,77.98,80.09,76.91,77.97,76.47,77.5,77.63,78.37,77.75,77.71,78.81,77.13,77.57,76.71,78.75,78.

22,78.51,NA,NA,NA,77.25,78.98,75.82,78.71,76.93,78.73,77.23,78.38,78.96,77.71,78.73,78.39,78.66

,77.77,79.57,76.93,79.72,76.21,76.63,78.03,77.5,78.08,76.84,NA,78.46,79.07,80.22,77,78.94,77.69,78 $.51,78.17,77.56,76.91,78.27$, NA,77.24,77.91,78.09,77.54,78.05,77.36,76.77,NA,NA,NA,NA,NA,79.0 $6,75.97,78.19,79.25,78.08,79.08,75.6,79.31,77.7,78.15, N A, N A, 78.21,78.23,79.84,78.75,77.52,76.55,7$ 6.66,79.68,77.3,NA,NA,NA,78.02,77.13,76.44,79.8,78.12,78.13,79.24,77.51,79.83,76.84,78.07,NA,7 7.33,78.29,79.02,80.36,80.13,77.98,76.92,76.01,78.42,77.23,78.6,76.94,78.21,78.84,78.22,77.47,77.5 8,78.48,77.55,77.98,NA,NA,NA,NA,78.55,77.16,77.08,78.5,77.54,78.88,78.97,NA,NA,NA,NA,NA, 7 8.22,77.72,80.33,77.9,79.03,78.97,79.24,78.59,78.19,79.52,77.85,78.05,78.67,76.2,77.72,78.51,76.58, 78.14,77.81,77.81,78.35,77.99,78.43,79.14,77.59,78.32,78.14,77.81,77.22,77.34,77.47,76.96,78.41,78 .46,NA,NA,79.84,78.99,78.54,78.21,78.64,76.22,78.62,78.54,78.08,NA,NA,NA,77.81,78.55,76.99,77. 96,78.96,77.99,77.43,78.12,NA,NA,NA,NA,77.21,78.9,78.96,76.86,77.91,78.47,78.34,NA,NA,NA,N A,NA,78.56,77.55,78.3,77.66,79.16,78.44,79.59,78.7,76.69,77.38,78.81,NA,80.19,77.3,76.57,80.58,7 7.64,77.09,77.93,79.13,76.99,77.68,78.72,76.41,78.65,77.4,77.7,77.45,77.71,77.55,76.06,79.77,NA,N A,NA,NA, 76.89,79.79,76.65,76.68,75.23,79.15,77.34,76.7,77.31,78.81,78.89,76.17,77.48,76.71,77.35 ,78.36,76.17,77.43,77.05,77.55,NA,NA,NA,NA,79.04,75.63,78.83,77.4,78.12,77.77,78.21,78.15,77.0 2,78.38,NA,NA,79.22,77.69,77.4,77.36,78.17,79.07,78.07,79.59,78.6,77.31,80.17,NA,78.72,79.75,78. $73,77.72,78.16,76.27,78.13,78.53,77.99,77.95, N A, N A, 79.11,77.87,77.98,77.1,78.2,78.25,78.32,78.18$, 
79.21,80.2,77.08,76.02,76.53,78.08,78.75,78.72,78.37,79.18,79.38,77.14,NA,NA,NA,NA,76.76,77.12 ,77.33,77.33,77.58,77.96,77.6,NA,NA,NA,NA,NA,78.71,77.08,77.58,79.29,77.79,77.69,78.54,77.26,7 $6.42,79.19, \mathrm{NA}, \mathrm{NA}), \mathrm{ncol}=12)$

Revenimento=matrix $(\mathrm{c}(74.29,73.43,75.14,76.39,75.3,75.66,75.52,74.81, N A, N A, N A, N A, 75.08,73.45$, 75.59,74.71,74.65,76.36,72.46,74.53,75.11,74.94,74.89,74.88,74.24,74.82,75.31,73.62,74.66,73.96,75 $.22,74.48,74.86, N A, N A, N A, 75.88,75.35,75.38,75.9,74.37,74.84,74.69,75.04,76.09,76.12,75.47,74.28$, $75.48,73.58,75.33,74.24,76.05,73.94,74.19,74.99,74.72,76.23,73.84, N A, 75.59,76.26,75.97,74.57,75.9$ $1,74.1,75.65,74.97,75.37,75.21,75.68, N A, 74.89,74.23,74.97,74.74,75.84,74.49,74.1, N A, N A, N A, N A$, NA,74.04,73.36,75.55,74.9,75.38,74.71,75.26,74.62,74.14,75.53,NA,NA,75.72,74.48,76.05,75.71,76. 68,73.62,74.9,73.93,74.63,NA,NA,NA,75.9,74.19,74.12,75.81,73.78,75.24,76.05,75.25,76.6,74.65,74 61,NA,75.05,76.16,75.2,76.37,76.1,74.21,75.16,73.87,74.71,74.47,76.4,75.63,74.63,76.08,75.63,74.77 ,74.35,74.93,74.12,74.39,NA,NA,NA,NA,76.38,75,74.7,75.88,75.34,75.55,75.64,NA,NA,NA,NA,NA ,75.6,74.98,77.59,76.45,75.77,76.09,75.21,76.38,74.55,74.77,74.13,74.58,75.03,74.68,73.91,75.35,73. $85,74.81,74.72,74.83,75.77,74.33,74.46,74.48,75.11,74.9,75.76,74.63,75.53,74.94,74,75.66,74.88,74$. 96,NA,NA,74.25,75.02,76.53,75.14,74.76,74.67,74.91,74.83,75.31,NA,NA,NA,75.42,75.22,74.48,75. 15,75.7,75.43,74,75.77,NA,NA,NA,NA, 74.25,73.98,74.84,75.34,76.43,74.33,74.81,NA,NA,NA,NA, NA,74.77,74.54,75.89,76.07,74.59,76.01,74.71,74.92,74.26,75.64,75.1,NA,73.91,74.76,74.7,74.3,74.7 4,74.22,74.85,74.5,74.33,73.45,74.98,75.23,75.87,73.99,76.48,73.53,74.48,75.19,74.19,74.74,NA,NA, NA,NA,75.36,74.79,73.65,74.11,73.77,76.75,74.2,73.72,73.93,76,74.99,75.46,74.21,74.44,75.13,74.8 1,74.72,75.58,75.4,74.89,NA,NA,NA,NA,75.13,74.24,74.81,75.9,75.38,74.68,75.13,74.48,74.75,76.1, NA,NA,74.7,74.82,73.93,74.59,75.07,75.02,74.24,74.91,75.88,74.56,76.53,NA,75.69,75.45,74.4,75.2 $1,75.93,74.1,74.82,75.59,75.14,73.5, N A, N A, 75.52,74.35,75.08,74.25,75.44,75.68,74.97,72.82,75.84,7$ $5.49,74.77,74.06,73.47,75.42,73.31,74.84,74.32,74.52,75.7,74.69, N A, N A, N A, N A, 73.58,73.9,74.39,7$ 3.81,75.75,74.04,74.24,NA,NA,NA,NA,NA,74.6,74.35,75.3,74.68,76.03,75.51,75.06,74.06,74.21,74.7 6,NA,NA),ncol=12)

\#NA indica informações não obtidas

q<-qcc(Têmpera,type="S")

q<-qcc(Têmpera,type="xbar")

q<-qcc(Revenimento,type="S")

q<-qcc(Revenimento,type="xbar")

Anexo 3 - Algoritmo computacional do software R para gerar o GCM T² de Hotelling com tamanho de amostra variável.

$\mathrm{x} 1<-\mathrm{c}(76.8,76.41,79.8,78.12,77.92,78.72,78.95,77.81)$

$\mathrm{y} 1<-\mathrm{c}(74.29,73.43,75.14,76.39,75.3,75.66,75.52,74.81)$

$\mathrm{x} 2<-\mathrm{c}(78.64,76.99,78.49,75.98,77.98,80.09,76.91,77.97,76.47,77.5,77.63,78.37)$

y2<-c(75.08,73.45,75.59,74.71,74.65,76.36,72.46,74.53,75.11,74.94,74.89,74.88)

$\mathrm{x} 3<-\mathrm{c}(77.75,77.71,78.81,77.13,77.57,76.71,78.75,78.22,78.51)$

$\mathrm{y} 3<-\mathrm{c}(74.24,74.82,75.31,73.62,74.66,73.96,75.22,74.48,74.86)$

$\mathrm{x} 4<-\mathrm{c}(77.25,78.98,75.82,78.71,76.93,78.73,77.23,78.38,78.96,77.71,78.73,78.39)$

$\mathrm{y} 4<-\mathrm{c}(75.88,75.35,75.38,75.9,74.37,74.84,74.69,75.04,76.09,76.12,75.47,74.28)$

$\mathrm{x} 5<-\mathrm{c}(78.66,77.77,79.57,76.93,79.72,76.21,76.63,78.03,77.5,78.08,76.84)$

$\mathrm{y} 5<-\mathrm{c}(75.48,73.58,75.33,74.24,76.05,73.94,74.19,74.99,74.72,76.23,73.84)$

x6<-c(78.46,79.07,80.22,77,78.94,77.69,78.51,78.17,77.56,76.91,78.27)

y6<-c $(75.59,76.26,75.97,74.57,75.91,74.1,75.65,74.97,75.37,75.21,75.68)$

$\mathrm{x} 7<-\mathrm{c}(77.24,77.91,78.09,77.54,78.05,77.36,76.77)$

$\mathrm{y} 7<-\mathrm{c}(74.89,74.23,74.97,74.74,75.84,74.49,74.1)$

$\mathrm{x} 8<-\mathrm{c}(79.06,75.97,78.19,79.25,78.08,79.08,75.6,79.31,77.7,78.15)$

$\mathrm{y} 8<-\mathrm{c}(74.04,73.36,75.55,74.9,75.38,74.71,75.26,74.62,74.14,75.53)$

$\mathrm{x} 9<-\mathrm{c}(78.21,78.23,79.84,78.75,77.52,76.55,76.66,79.68,77.3)$

$\mathrm{y} 9<-\mathrm{c}(75.72,74.48,76.05,75.71,76.68,73.62,74.9,73.93,74.63)$

x10<-c $(78.02,77.13,76.44,79.8,78.12,78.13,79.24,77.51,79.83,76.84,78.07)$

$\mathrm{y} 10<-\mathrm{c}(75.9,74.19,74.12,75.81,73.78,75.24,76.05,75.25,76.6,74.65,74.61)$

$\mathrm{x} 11<-\mathrm{c}(77.33,78.29,79.02,80.36,80.13,77.98,76.92,76.01,78.42,77.23,78.6,76.94)$

y11<-c $(75.05,76.16,75.2,76.37,76.1,74.21,75.16,73.87,74.71,74.47,76.4,75.63)$ 
$\mathrm{x} 12<-\mathrm{c}(78.21,78.84,78.22,77.47,77.58,78.48,77.55,77.98)$

y12<-c $(74.63,76.08,75.63,74.77,74.35,74.93,74.12,74.39)$

$\mathrm{x} 13<-\mathrm{c}(78.55,77.16,77.08,78.5,77.54,78.88,78.97)$

y13<-c $(76.38,75,74.7,75.88,75.34,75.55,75.64)$

$\mathrm{x} 14<-\mathrm{c}(78.22,77.72,80.33,77.9,79.03,78.97,79.24,78.59,78.19,79.52,77.85,78.05)$

y14<-c (75.6,74.98,77.59,76.45,75.77,76.09,75.21,76.38,74.55,74.77,74.13,74.58)

$\mathrm{x} 15<-\mathrm{c}(78.67,76.2,77.72,78.51,76.58,78.14,77.81,77.81,78.35,77.99,78.43,79.14)$

y15<-c $(75.03,74.68,73.91,75.35,73.85,74.81,74.72,74.83,75.77,74.33,74.46,74.48)$

$\mathrm{x} 16<-\mathrm{c}(77.59,78.32,78.14,77.81,77.22,77.34,77.47,76.96,78.41,78.46)$

y16<-c(75.11,74.9,75.76,74.63,75.53,74.94,74,75.66,74.88,74.96)

$\mathrm{x} 17<-\mathrm{c}(79.84,78.99,78.54,78.21,78.64,76.22,78.62,78.54,78.08)$

$\mathrm{y} 17<-\mathrm{c}(74.25,75.02,76.53,75.14,74.76,74.67,74.91,74.83,75.31)$

$\mathrm{x} 18<-\mathrm{c}(77.81,78.55,76.99,77.96,78.96,77.99,77.43,78.12)$

y18<-c $(75.42,75.22,74.48,75.15,75.7,75.43,74,75.77)$

$\mathrm{x} 19<-\mathrm{c}(77.21,78.9,78.96,76.86,77.91,78.47,78.34)$

y19<-c (74.25,73.98,74.84,75.34,76.43,74.33,74.81)

$\mathrm{x} 20<-\mathrm{c}(78.56,77.55,78.3,77.66,79.16,78.44,79.59,78.7,76.69,77.38,78.81)$

y20<-c $(74.77,74.54,75.89,76.07,74.59,76.01,74.71,74.92,74.26,75.64,75.1)$

x21<-c $(80.19,77.3,76.57,80.58,77.64,77.09,77.93,79.13,76.99,77.68,78.72,76.41)$

y21<-c(73.91,74.76,74.7,74.3,74.74,74.22,74.85,74.5,74.33,73.45,74.98,75.23)

$\mathrm{x} 22<-\mathrm{c}(78.65,77.4,77.7,77.45,77.71,77.55,76.06,79.77)$

y22<-c(75.87,73.99,76.48,73.53,74.48,75.19,74.19,74.74)

$\mathrm{x} 23<-\mathrm{c}(76.89,79.79,76.65,76.68,75.23,79.15,77.34,76.7,77.31,78.81,78.89,76.17)$

y23<-c(75.36,74.79,73.65,74.11,73.77,76.75,74.2,73.72,73.93,76,74.99,75.46)

x $24<-c(77.48,76.71,77.35,78.36,76.17,77.43,77.05,77.55)$

y24<-c(74.21,74.44,75.13,74.81,74.72,75.58,75.4,74.89)

$\mathrm{x} 25<-\mathrm{c}(79.04,75.63,78.83,77.4,78.12,77.77,78.21,78.15,77.02,78.38)$

y $25<-c(75.13,74.24,74.81,75.9,75.38,74.68,75.13,74.48,74.75,76.1)$

$\mathrm{x} 26<-\mathrm{c}(79.22,77.69,77.4,77.36,78.17,79.07,78.07,79.59,78.6,77.31,80.17)$

y26<-c (74.7,74.82,73.93,74.59,75.07,75.02,74.24,74.91,75.88,74.56,76.53)

$\mathrm{x} 27<-\mathrm{c}(78.72,79.75,78.73,77.72,78.16,76.27,78.13,78.53,77.99,77.95)$

y27<-c $(75.69,75.45,74.4,75.21,75.93,74.1,74.82,75.59,75.14,73.5)$

$\mathrm{x} 28<-\mathrm{c}(79.11,77.87,77.98,77.1,78.2,78.25,78.32,78.18,79.21,80.2,77.08,76.02)$

y $28<-c(75.52,74.35,75.08,74.25,75.44,75.68,74.97,72.82,75.84,75.49,74.77,74.06)$

$\mathrm{x} 29<-\mathrm{c}(76.53,78.08,78.75,78.72,78.37,79.18,79.38,77.14)$

y29<-c $(73.47,75.42,73.31,74.84,74.32,74.52,75.7,74.69)$

$\mathrm{x} 30<-\mathrm{c}(76.76,77.12,77.33,77.33,77.58,77.96,77.6)$

y30<-c $(73.58,73.9,74.39,73.81,75.75,74.04,74.24)$

$\mathrm{x} 31<-\mathrm{c}(78.71,77.08,77.58,79.29,77.79,77.69,78.54,77.26,76.42,79.19)$

y31<-c(74.6,74.35,75.3,74.68,76.03,75.51,75.06,74.06,74.21,74.76)

$\mathrm{x}=\mathrm{c}(\mathrm{x} 1, \mathrm{x} 2, \mathrm{x} 3, \mathrm{x} 4, \mathrm{x} 5, \mathrm{x} 6, \mathrm{x} 7, \mathrm{x} 8, \mathrm{x} 9, \mathrm{x} 10, \mathrm{x} 11, \mathrm{x} 12, \mathrm{x} 13, \mathrm{x} 14, \mathrm{x} 15, \mathrm{x} 16, \mathrm{x} 17, \mathrm{x} 18, \mathrm{x} 19, \mathrm{x} 20, \mathrm{x} 21, \mathrm{x} 22, \mathrm{x} 23, \mathrm{x} 24, \mathrm{x} 25$, $\mathrm{x} 26, \mathrm{x} 27, \mathrm{x} 28, \mathrm{x} 29, \mathrm{x} 30, \mathrm{x} 31)$

$\mathrm{y}=\mathrm{c}(\mathrm{y} 1, \mathrm{y} 2, \mathrm{y} 3, \mathrm{y} 4, \mathrm{y} 5, \mathrm{y} 6, \mathrm{y} 7, \mathrm{y} 8, \mathrm{y} 9, \mathrm{y} 10, \mathrm{y} 11, \mathrm{y} 12, \mathrm{y} 13, \mathrm{y} 14, \mathrm{y} 15, \mathrm{y} 16, \mathrm{y} 17, \mathrm{y} 18, \mathrm{y} 19, \mathrm{y} 20, \mathrm{y} 21, \mathrm{y} 22, \mathrm{y} 23, \mathrm{y} 24, \mathrm{y} 25$, $\mathrm{y} 26, \mathrm{y} 27, \mathrm{y} 28, \mathrm{y} 29, \mathrm{y} 30, \mathrm{y} 31)$

\#Verificando a correlação entre as variáveis têmpera e revenimento e a suposição de normalidade bivariada

cor.test $(\mathrm{x}, \mathrm{y})$

install.packages ("mvnormtest")

library(mvnormtest)

vetor=matrix $(\mathrm{c}(\mathrm{x}, \mathrm{y}), \mathrm{ncol}=2)$

vetor $1=\mathrm{t}($ vetor $)$

vetor<-matrix $(\mathrm{t}(\mathrm{c}(\mathrm{x}, \mathrm{y})), 2$, length $(\mathrm{x}))$

mshapiro.test(vetor1)

\#Entrar com a média de cada instante de cada característica da qualidade 
media1=matrix(c(mean(x1), mean(x2), mean(x3), mean(x4), mean(x5), mean(x6), mean(x7), mean(x8), me $\operatorname{an}(x 9)$, mean(x10), mean(x11), mean(x12), mean(x13), mean(x14), mean(x15), mean(x16), mean(x17), mea $\mathrm{n}(\mathrm{x} 18)$, mean(x19), mean(x20), mean(x21), mean(x22), mean(x23), mean(x24), mean(x25), mean(x26), me $\operatorname{an}(x 27)$, mean(x28), mean(x29), mean(x30), mean(x31)))

media2=matrix $(\mathrm{c}($ mean $(\mathrm{y} 1)$, mean $(\mathrm{y} 2)$, mean $(\mathrm{y} 3)$, mean $(\mathrm{y} 4)$, mean $(\mathrm{y} 5)$, mean $(\mathrm{y} 6)$, mean $(\mathrm{y} 7)$, mean $(\mathrm{y} 8)$, ,me an(y9), mean(y10), mean(y11), mean(y12), mean(y13), mean(y14), mean(y15), mean(y16), mean(y17), mea n(y18), mean(y19), mean(y20), mean(y21), mean(y22), mean(y23), mean(y24), mean(y25), mean(y26), me an(y27), mean(y28), mean(y29), mean(y30), mean(y31)))

\#Entrar com a variância de cada instante de cada característica da qualidade

variancia1 = matrix (c(var(x1), $\operatorname{var}(\mathrm{x} 2), \operatorname{var}(\mathrm{x} 3), \operatorname{var}(\mathrm{x} 4), \operatorname{var}(\mathrm{x} 5), \operatorname{var}(\mathrm{x} 6), \operatorname{var}(\mathrm{x} 7), \operatorname{var}(\mathrm{x} 8), \operatorname{var}(\mathrm{x} 9), \operatorname{var}(\mathrm{x} 10)$, $\operatorname{var}(\mathrm{x} 11), \operatorname{var}(\mathrm{x} 12), \mathrm{var}(\mathrm{x} 13), \operatorname{var}(\mathrm{x} 14), \operatorname{var}(\mathrm{x} 15), \mathrm{var}(\mathrm{x} 16), \operatorname{var}(\mathrm{x} 17), \operatorname{var}(\mathrm{x} 18), \operatorname{var}(\mathrm{x} 19), \operatorname{var}(\mathrm{x} 20), \operatorname{var}(\mathrm{x} 21), \mathrm{v}$ $\operatorname{ar}(x 22), \operatorname{var}(x 23), \operatorname{var}(x 24), \operatorname{var}(x 25), \operatorname{var}(x 26), \operatorname{var}(x 27), \operatorname{var}(x 28), \operatorname{var}(x 29), \operatorname{var}(x 30), \operatorname{var}(x 31)))$ variancia2=matrix $(c(\operatorname{var}(y 1), \operatorname{var}(y 2), \operatorname{var}(y 3), \operatorname{var}(y 4), \operatorname{var}(y 5), \operatorname{var}(y 6), \operatorname{var}(y 7), \operatorname{var}(y 8), \operatorname{var}(y 9), \operatorname{var}(y 10)$, $\operatorname{var}(\mathrm{y} 11), \operatorname{var}(\mathrm{y} 12), \operatorname{var}(\mathrm{y} 13), \operatorname{var}(\mathrm{y} 14), \operatorname{var}(\mathrm{y} 15), \operatorname{var}(\mathrm{y} 16), \operatorname{var}(\mathrm{y} 17), \operatorname{var}(\mathrm{y} 18), \operatorname{var}(\mathrm{y} 19), \operatorname{var}(\mathrm{y} 20), \operatorname{var}(\mathrm{y} 21), \mathrm{v}$ $\operatorname{ar}(\mathrm{y} 22), \operatorname{var}(\mathrm{y} 23), \operatorname{var}(\mathrm{y} 24), \operatorname{var}(\mathrm{y} 25), \operatorname{var}(\mathrm{y} 26), \operatorname{var}(\mathrm{y} 27), \operatorname{var}(\mathrm{y} 28), \operatorname{var}(\mathrm{y} 29), \operatorname{var}(\mathrm{y} 30), \operatorname{var}(\mathrm{y} 31)))$

\#Calcular a covariância de cada instante das características da qualidade

$\operatorname{covar}=\operatorname{matrix}(\mathrm{c}(\operatorname{cov}(\mathrm{x} 1, \mathrm{y} 1), \operatorname{cov}(\mathrm{x} 2, \mathrm{y} 2), \operatorname{cov}(\mathrm{x} 3, \mathrm{y} 3), \operatorname{cov}(\mathrm{x} 4, \mathrm{y} 4), \operatorname{cov}(\mathrm{x} 5, \mathrm{y} 5), \operatorname{cov}(\mathrm{x} 6, \mathrm{y} 6), \operatorname{cov}(\mathrm{x} 7, \mathrm{y} 7), \operatorname{cov}(\mathrm{x}$ $8, \mathrm{y} 8), \operatorname{cov}(\mathrm{x} 9, \mathrm{y} 9), \operatorname{cov}(\mathrm{x} 10, \mathrm{y} 10), \operatorname{cov}(\mathrm{x} 11, \mathrm{y} 11), \operatorname{cov}(\mathrm{x} 12, \mathrm{y} 12), \operatorname{cov}(\mathrm{x} 13, \mathrm{y} 13), \operatorname{cov}(\mathrm{x} 14, \mathrm{y} 14), \operatorname{cov}(\mathrm{x} 15, \mathrm{y} 15), \mathrm{c}$ ov $(\mathrm{x} 16, \mathrm{y} 16), \operatorname{cov}(\mathrm{x} 17, \mathrm{y} 17), \operatorname{cov}(\mathrm{x} 18, \mathrm{y} 18), \operatorname{cov}(\mathrm{x} 19, \mathrm{y} 19), \operatorname{cov}(\mathrm{x} 20, \mathrm{y} 20), \operatorname{cov}(\mathrm{x} 21, \mathrm{y} 21), \operatorname{cov}(\mathrm{x} 22, \mathrm{y} 22), \operatorname{cov}(\mathrm{x}$ $23, \mathrm{y} 23), \operatorname{cov}(\mathrm{x} 24, \mathrm{y} 24), \operatorname{cov}(\mathrm{x} 25, \mathrm{y} 25), \operatorname{cov}(\mathrm{x} 26, \mathrm{y} 26), \operatorname{cov}(\mathrm{x} 27, \mathrm{y} 27), \operatorname{cov}(\mathrm{x} 28, \mathrm{y} 28), \operatorname{cov}(\mathrm{x} 29, \mathrm{y} 29), \operatorname{cov}(\mathrm{x} 30, \mathrm{y}$ $30), \operatorname{cov}(\mathrm{x} 31, \mathrm{y} 31)))$

\#Calcular a média das médias de cada característica da qualidade e criar uma matriz com os resultados xbarbar1=mean(media1)

$\mathrm{xbarbar} 2=$ mean $($ media 2$)$

xbarbar=matrix $(\mathrm{c}(\mathrm{xbarbar} 1, \mathrm{xbarbar2}), 2,1)$

\#Calcular a média das variâncias e covariâncias das características da qualidade

medvar $1=$ mean $($ variancia 1$)$

medvar $2=$ mean $($ variancia 2$)$

medcov=mean (covar)

\#Criar a matriz de variâncias e covariâncias

$S<-$ matrix $(0,2,2)$

S[1,1]<-medvar1 \#Entrar com a média das variâncias da variável 1

$\mathrm{S}[1,2]<-$ medcov \#Entrar com a média das covariâncias das variáveis 1 e 2

$\mathrm{S}[2,1]<-$ medcov \#Entrar com a média das covariâncias das variáveis 2 e 1

$\mathrm{S}[2,2]<-$-medvar2 \#Entrar com a média das variâncias da variável 2

\#Tamanho amostral de cada instante

$\mathrm{n}=\mathrm{c}(8,12,9,12,11,11,7,10,9,11,12,9,7,12,12,10,9,8,7,11,12,8,12,8,10,11,10,12,8,7,10)$

$\mathrm{p}=2 \#$ Número de características da qualidade avaliadas

$\mathrm{m}=31$ \#Número de amostras

$\mathrm{v}=$ matrix $(\mathrm{c}$ (media1, media2),m,2) \#Vetor de médias das amostras

$\mathrm{r}=\mathrm{t}(\mathrm{v})$ \#Transposta de $\mathrm{v}$

\#Estatística $\mathrm{T}^{2}$ de Hotelling

$\mathrm{T} 2=$ matrix $(0, \mathrm{~m}, 1)$

for $(\mathrm{i}$ in $1: \mathrm{m})\{\mathrm{T} 2[\mathrm{i}]=\mathrm{n}[\mathrm{i}] * \mathrm{t}(\mathrm{r}[, \mathrm{i}]-\mathrm{xbarbar}) \% * \% \operatorname{solve}(\mathrm{S}) \% * \%(\mathrm{r}[\mathrm{i}]-\mathrm{xbarbar})\}$

\#Quantil da Distribuição $\mathrm{F}$

$\mathrm{qF}=\operatorname{matrix}(0, \mathrm{~m}, 1)$

for $(\mathrm{i}$ in $1: \mathrm{m})\{\mathrm{qF}[\mathrm{i}]=\mathrm{qf}(0.95, \mathrm{p},((\mathrm{m} * \mathrm{n}[\mathrm{i}])-\mathrm{m}-\mathrm{p}+1))\}$

\# Calcular o Limite de Controle, com um grau de confiança igual a 0,95

lsck=matrix $(0, \mathrm{~m}, 1)$

for $(\mathrm{i}$ in $1: \mathrm{m})\{1 \mathrm{sck}[\mathrm{i}]=((\mathrm{p} *(\mathrm{~m}-1) *(\mathrm{n}[\mathrm{i}]-1)) /((\mathrm{m} * \mathrm{n}[\mathrm{i}])-\mathrm{m}-\mathrm{p}+1)) * \mathrm{qF}[\mathrm{i}]\}$

$\mathrm{x}<-\mathrm{c}(1: 31)$ \#Amostras

$\mathrm{y}=\mathrm{c}(\mathrm{T} 2)$ \#Estatística $\mathrm{T} 2$ de Hotelling

$\mathrm{z}=1$ sck \#Limite superior de controle do gráfico T2

plot(x,y,type="b",xlab="Dias",ylab="T2")

lines $(\mathrm{x}, \mathrm{z})$ \#Só depois de gerar o gráfico copie e cole os comandos abaixo 
points $(14,8.728823$, col = "black", pch=19)

points (30, 6.345474, col = " black ", pch=19)

\#Decomposição dos pontos fora de controle

Vtx14<-c(mean $(\mathrm{x} 14))$

Vty14<-c(mean $(\mathrm{y} 14))$

$\mathrm{Vtx} 30<-\mathrm{c}(\operatorname{mean}(\mathrm{x} 30))$

$\operatorname{Vty} 30<-\mathrm{c}(\operatorname{mean}(\mathrm{y} 30))$

T141<-12*t(Vty14-xbarbar2)\%*\%solve(medvar2)\%*\%(Vty14-xbarbar2)

T142<-12*t(Vtx14-xbarbar1)\%*\%solve(medvar1)\%*\%(Vtx14-xbarbar1)

T301<-7*t(Vty30-xbarbar2)\%*\%solve(medvar2)\%*\%(Vty30-xbarbar2)

T302<-7*t(Vtx30-xbarbar1)\%*\%solve(medvar1)\%*\%(Vtx30-xbarbar1)

d1T141<-(T2[14]-T141)

d2T142<-(T2[14]-T142)

d1T301<-(T2[30]-T301)

d2T302<-(T2[30]-T302) 